\title{
I distretti industriali italiani: recenti tendenze evolutive
}

\author{
ANTONIO RICCIARDI
}

\begin{abstract}
Obiettivo del paper: Verificare se gli effetti della recente crisi sono stati mitigati, nel caso dei distretti industriali, dalle loro peculiarità organizzative e dalle rispettive competenze distintive.

Metodologia: Il paper propone una nuova classificazione dei distretti industriali (dinamici, maturi, vulnerabili, virtuali) e per ciascuna categoria, anche con ausilio di casi, si cerca di individuare le performance, i fattori critici di successo/insuccesso, l'impatto della crisi e le rispettive "reazioni" strategiche.

Risultati: I vantaggi localizzativi di cui godevano le imprese distrettuali non sono scomparsi ovunque - vi sono alcuni distretti che registrano ancora oggi performance di gran lunga superiori alla media del settore - e comunque ciò che si rileva positivamente nei numerosi sistemi locali industriali sono la capacità di anticipare con scelte strategiche opportune le tendenze future e le profonde trasformazioni organizzative come risposta alle dinamiche discontinue della congiuntura.

Limiti della ricerca: La classificazione dei distretti proposta si basa in parte sull'analisi ed elaborazione di variabili qualitative

Implicazioni pratiche: In uno scenario di mercato globalizzato, il ruolo del territorio paradossalmente diventa ancora più importante per sostenere la stessa sopravvivenza dei distretti. C'è bisogno, tuttavia, di un territorio «attrezzato», che metta a disposizione delle imprese conoscenze, technicalities avanzate, servizi finanziari innovativi.

Originalità del lavoro: La classificazione dei distretti è originale e non risultano in letteratura studi recenti sull'analisi dell'impatto della crisi sulle performance dei distretti e sulle strategie messe in atto per fronteggiarla.
\end{abstract}

Parole chiave: classificazione distretti industriali; impatto della crisi e reazione strategica dei distretti; reti di imprese; reti tra distretti

Purpose of the paper: To verify whether the effects of the recent crisis have been mitigated in the case of industrial districts by their organizational peculiarities and distinctive competencies.

Methodology: The paper proposes, through the analysis of qualitative variables, a new classification of industrial districts (dynamic, mature, vulnerable, virtual), and for each

Ordinario di Economia Aziendale - Università degli Studi della Calabria

e-mail: antonio.ricciardi@unical.it

sinergie, rivista di studi e ricerche

n. 91, Maggio-Agosto 2013, p. 21-58

ISSN 0393-5108 - DOI 10.7433/s91.2013.03 
category, with the aid of case studies, it seeks to identify the performance, the critical factors of success / failure, and the impact of the crisis and their strategic "reaction".

Findings: The location advantage enjoyed by the district firms has not disappeared anywhere: there are some districts that still, today, record performance far superior to the industry average, and a lot of local industrial systems are able to anticipate future trends with appropriate strategic choices and adopt organizational transformations in order to face the dynamics of the economic trend.

Research limits: The classification of the industrial districts, are based on the analysis and processing of qualitative variables.

Practical implications: In a globalized market, the role of the territory paradoxically becomes even more important going as far as to support the continued survival of the districts. There is a need, however, for a territory to be "equipped" that provides business knowledge, advanced technical knowledge, and innovative financial services.

Originality of the study: The classification of districts is original and does not appear in recent studies of the analysis of the impact of the crisis on the districts' performance and the strategies adopted to face it.

Key words:classification of industrial districts; the impact of the crisis and strategic reaction of the districts; business networks; networks between districts

\section{I distretti: definizione, caratteristiche delle imprese distrettuali e vantaggi competitivi}

Il distretto industriale, modello organizzativo tipico dell'economia italiana, è un'area territoriale con un'alta concentrazione di piccole e medie imprese industriali ad elevata specializzazione produttiva, generalmente caratterizzate da un'intensa interdipendenza dei loro cicli produttivi e fortemente integrate con l'ambiente socioeconomico locale che le ospita.

Il distretto industriale non va confuso con le aree industriali: territori dove sono concentrate imprese di differenti settori e specializzazioni, molto spesso senza aver sviluppato tra loro alcun tipo di collaborazione e senza alcun legame con il territorio.

Generalmente in un distretto operano imprese indipendenti, integrate in una rete di relazioni di cooperazione informale e di lungo periodo. Da questo punto di vista, il distretto rappresenta indubbiamente un terreno fertile per il sorgere di un sistema reticolare, tenuto conto che si innesta in un tessuto di relazioni (anche sociali) preesistenti in aree geograficamente ben delimitate. La caratteristica peculiare dei distretti, soprattutto quelli più dinamici, è, infatti, la collaborazione tra imprese: si distribuiscono gli ordini di produzione; realizzano insieme servizi; mettono in comune conoscenze e sviluppano insieme innovazione tecnologica ${ }^{1}$.

1 Un esempio emblematico di cooperazione tra aziende è offerto dal distretto conciario di Santa Croce - Pisa (1400 aziende, 10.000 addetti, 2,6 miliardi di fatturato, 40\% export) dove le imprese con investimenti comuni hanno realizzato un Centro di ricerca con l'Università di Pisa per ridurre l'emissione di gas inquinanti. Il risultato di questa collaborazione è stata la depurazione del $98 \%$ del carico inquinante contro il $70 \%$ della 
I distretti industriali rappresentano un modello organizzativo tipico dell'economia italiana che il mondo studia e cerca di imitare. L'ultimo censimento Istat (2001) ha rilevato l'esistenza di 156 distretti (12,5 milioni di abitanti; 22,2\% della popolazione) la maggior parte dei quali localizzati nel Centro Italia (49) e nel nord est (42) come illustrato nella Figura $1^{2}$.

Fig. 1: Distretti industriali per area geografica

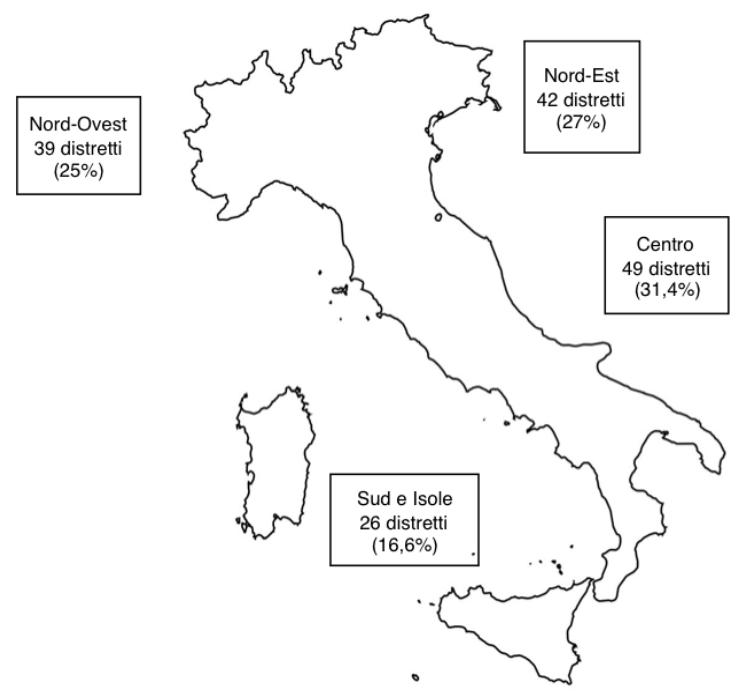

Fonte: nostra elaborazione su dati Istat (Censimento 2001)

Le 215mila aziende distrettuali con circa 2 milioni di addetti (39,3\% del settore manifatturiero) realizzano il $27,2 \%$ del Pil e il $37,2 \%$ delle esportazioni, con quote superiori al $50 \%$ in alcuni settori di eccellenza ${ }^{3}$.

In termini di composizione settoriale, il sistema distrettuale italiano è fondamentalmente basato sulle attività produttive tipiche del Made in Italy, come messo in evidenza dalla numerosità delle imprese operanti nei settori del tessile-

media mondiale. Queste aziende, insieme, oltre ad esportare prodotti, esportano anche tecnologia di cui la Cina è uno dei principali Paesi importatori.

2 La rilevanza dei distretti è maggiore nel Centro Nord $(43 \%$ dell'occupazione manifatturiera dell'area) mentre è limitata nel Mezzogiorno (la stessa quota scende al $10 \%$ ). Il fenomeno distrettuale è assente in Valle d'Aosta e Liguria mentre ha la maggiore diffusione nelle Marche dove 1'80\% degli occupati manifatturieri e il 70\% dell'export si rilevano nei distretti (cfr. Cap.3 del Terzo Rapporto dell'Osservatorio Nazionale Distretti Italiani).

3 Tessile-abbigliamento $67 \%$, cuoio e prodotti in cuoio $66,9 \%$, lavorazioni dei minerali non metalliferi (incluse piastrelle e pietre ornamentali) $60,4 \%$, legno e prodotti in legno (esclusi mobili) 55,8\%, macchine e apparecchi 51,6\%, metalli e prodotti in metallo $51 \%$. 
abbigliamento $(28,8 \%)$, della meccanica tradizionale $(24,4 \%)$, dell'arredo-casa $(20,5 \%)$, della pelletteria e delle calzature $(12,8 \%)$. Questi settori sono presenti in 135 distretti (l'86,5\% del totale), cui si aggiungono quelli degli elettrodomestici, della meccanica strumentale, del packaging ed imballaggio e della produzione agroalimentare.

La maggior parte dei distretti italiani è nata e si è ampliata durante il miracolo economico degli anni Cinquanta e Sessanta, quando l'economia cresceva a ritmi elevati. Hanno poi conosciuto un significativo sviluppo negli anni Settanta e Ottanta, quando il sistema economico italiano ha rallentato la corsa mentre i distretti hanno portato sviluppo e benessere in aree allora arretrate come il Nord-Est e le regioni centrali lungo la costa adriatica. Negli anni Ottanta e soprattutto negli anni Novanta i distretti industriali hanno cominciato a prendere piede anche nel Sud del Paese.

Ciò che ha contraddistinto lo sviluppo e il successo della maggior parte dei distretti è stata la spontaneità dell'iniziativa promossa dal basso, dagli stessi imprenditori che nel corso degli anni hanno sviluppato competenze specialistiche in determinati settori e fondato la loro crescita sulle relazioni informali con le altre imprese e con il territorio. La spontaneità che contraddistingue il processo di addensamento dei distretti e la naturalezza con cui si instaurano le connessioni tra aziende operanti in una certa area, sembrano escludere a priori la possibilità di replicare in altre zone, attraverso politiche industriali preordinate e intenzionali, tali esperienze. La verifica empirica conferma questa considerazione ${ }^{4}$.

Le imprese localizzate nei distretti assumono caratteristiche diverse in base ad alcune variabili come l'autonomia strategica, lo sviluppo di competenze distintive nelle diverse aree della gestione e il grado di stabilità delle aziende.

In base a queste variabili è possibile individuare quattro profili di imprese distrettuali (Fig. 2) (Ricciardi, 2003; Izzo e Ricciardi, 2006; Ricciardi, 2008-a; Onida et al., 1992; Viesti, 2000).

Le imprese «bloccate» sono di piccole e piccolissime dimensioni (laboratori artigiani), specializzate in singole fasi di lavorazione, a basso contenuto professionale, in grado di garantire bassi costi ed adeguati standard qualitativi ma incapaci di esprimere in autonomia scelte strategiche idonee ad un inserimento autonomo nel mercato. Deboli sotto il profilo contrattuale, queste imprese risultano strettamente dipendenti dalle imprese committenti e più di altre stanno subendo gli effetti della crisi registrando percentuali più alte di chiusure e fallimenti.

Le imprese «trainate» presidiano le funzioni critiche della produzione, anche ricorrendo alla rete di fornitori locali, ma come le imprese bloccate assumono un comportamento adattivo-imitativo soprattutto nei confronti dell'innovazione di

4 Il patrimonio relazionale, ossia il vantaggio competitivo primario di ogni distretto industriale, dal quale scaturiscono tutti gli altri, difficilmente potrebbe essere ripristinato una volta distrutto, né potrebbe essere artificialmente costituito, per via esogena, in contesti in cui non emerge spontaneamente. Una politica di sviluppo può quindi contribuire a rafforzare i caratteri di una comunità ma non è sufficiente a costruire la comunità (Bellandi e Russo, 1994). 
prodotto e di processo. Per limiti della loro organizzazione non riescono a cogliere direttamente le opportunità della crescita export e, pur impegnate a migliorare la qualità dei prodotti, sostanzialmente la loro strategia competitiva si risolve nello sfruttare le condizioni facilitanti offerte dal contesto distrettuale ma quando queste vengono meno sono fortemente esposte alla concorrenza di fornitori esteri con più basso costo del lavoro.

Fig. 2: Profili delle imprese distrettuali

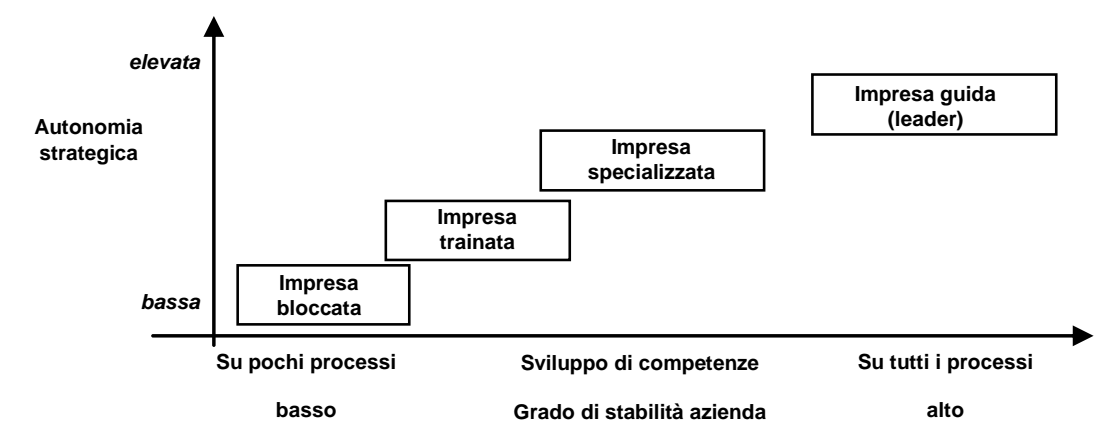

Fonte: ns. elaborazione

Le imprese «specializzate» focalizzate su elevate competenze produttive (progettazione e ingegnerizzazione del prodotto), sono capaci di realizzare, anche avvalendosi di sub-fornitori specializzati, semilavorati e prodotti finiti ad alto contenuto tecnologico ma difficilmente con marchio proprio. Si tratta di imprese che generalmente svolgono internamente poche fasi del processo produttivo, orientate allo sviluppo in proprio di innovazioni tecnologiche e di know-how esclusivo, ma che si rivelano deboli sul piano commerciale. Per questo motivo tendono a stabilire legami stretti con le imprese committenti (imprese leader), con la conseguenza che l'autonomia nella formulazione della propria strategia competitiva risulta essere condizionata dal "peso" che le produzioni in conto terzi assumono rispetto a quelle realizzate in proprio e con propri marchi. Come reazione alla crisi, queste aziende, sfruttando le competenze ad alta tecnologia, tendono ad occupare all'interno della filiera un segmento più remunerativo e con più alte barriere all'entrata, che le consentono di mettersi al riparo dalla concorrenza di subfornitori stranieri. In alcuni distretti, quelli più dinamici, le imprese specializzate, grazie ad una più elevata proiezione internazionale, hanno ampliato il numero dei committenti, in particolare aziende multinazionali che hanno favorito l'attività innovativa di queste imprese svolgendo il ruolo di trasmettitori di conoscenza tecnologica e manageriale. Le imprese specializzate rappresentano uno dei punti di forza dell'organizzazione distrettuale.

In alcuni distretti emergono alcune imprese di livello superiore (imprese leader/guida), non necessariamente di dimensioni superiori rispetto alle altre, ma maggiormente strutturate e che in genere sono presenti in maniera diretta sui mercati di sbocco, anche internazionali, con conoscenze tecnologiche ed un maggior potere 
contrattuale. Si tratta di aziende, spesso con marchi propri affermati sia sul mercato nazionale che internazionale, dotate di forte autonomia nell'elaborare le proprie scelte di posizionamento sui mercati di sbocco, in grado di svolgere al proprio interno tutte le fasi del processo produttivo, con competenze distintive soprattutto nella funzione ricerca e sviluppo e commerciale. Le imprese guida accentrano le fasi strategiche del processo produttivo mentre decentrano le fasi meramente tecnicoproduttive ad altre imprese (terzisti, laboratori artigiani, lavoranti a domicilio) sia interne che esterne al distretto. In sostanza, esse fungono da forze «motrici» dello sviluppo locale e svolgono un importante ruolo di interfaccia tra il mercato ed il sistema locale: attivano - quasi spontaneamente - la distribuzione tecnica del lavoro fra le unità operative interne, assumono il coordinamento dell'intera filiera produttiva distrettuale e ne promuovono politiche di marchio e di immagine ${ }^{5}$.

La competitività dei distretti industriali deriva dalla stessa organizzazione produttiva: la scomposizione (spaziale e temporale) del ciclo produttivo in fasi di lavorazioni e la specializzazione nell'esecuzione di ciascuna fase produttiva assicurano competitività sui costi, elevati livelli di flessibilità e capacità di innovazione (Fig. 3). La specializzazione delle fasi produttive permette il conseguimento di economie di scala e di apprendimento che riducono i costi unitari e favoriscono l'aumento della produttività.

Fig. 3: I vantaggi competitivi dei distretti

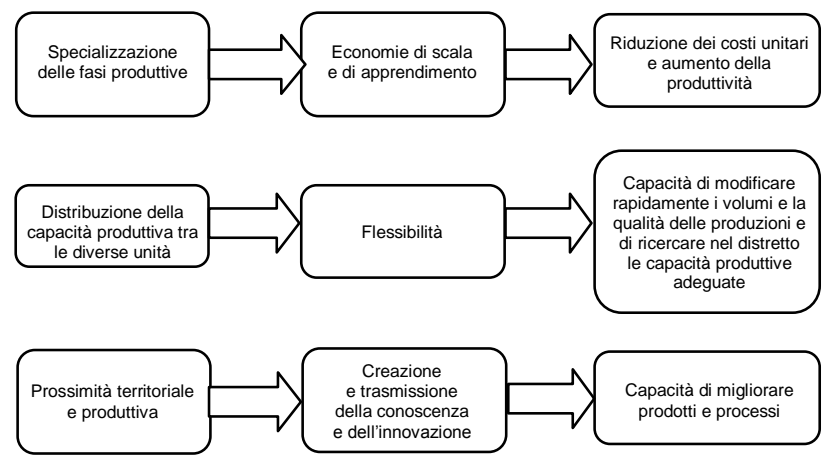

Fonte: ns. elaborazione

5 Liu Jo, azienda di produzione di abbigliamento fashion made in Italy, localizzata nel distretto tessile di Carpi, rappresenta un caso emblematico di azienda leader. Il brand, nato nel 1995, lancia inizialmente una collezione di maglieria per donna, per concentrarsi in seguito sulla caratterizzazione del marchio, rivolto ad un pubblico femminile selezionato. Prima produceva tutto all'interno, ora la produzione è tutta esternalizzata grazie ad una rete di fornitori coordinata da Liu-Jo. Attualmente, l'azienda si occupa solo di alcune fasi del processo produttivo: design, promozione del marchio, controllo dei canali di vendita. Grazie a questa strategia, attualmente i punti vendita monomarca sono circa 190 (di cui 120 all'estero) mentre sono 4.500 i clienti multimarca; il fatturato 2010 è stato pari a $230 \mathrm{mln}$ di euro (91 $\mathrm{mln}$ nel $2005,+152 \%$ in quattro anni). 
La distribuzione della capacità produttiva tra le diverse unità consente di realizzare elevati livelli di flessibilità. Tale flessibilità va intesa sia in termini di capacità di modificare rapidamente i volumi e la qualità delle produzioni (con costi analoghi - o inferiori - a quelli delle produzioni standardizzate) sia in termini di possibilità di ricercare nel distretto la risposta più opportuna tra le capacità produttive diffuse e disponibili. Il fatto che nel distretto siano presenti risorse di lavoro specializzate, dotate di un'elevata mobilità interna fra un'impresa e l'altra consente, per esempio, di intensificare il ricorso alla sub-fornitura e al lavoro a domicilio in presenza di aumenti anche transitori dei livelli produttivi. Ciò conferisce dinamicità al distretto industriale nel suo complesso e rappresenta il suo punto di forza nella competizione internazionale.

La prossimità, territoriale e produttiva, delle imprese favorisce processi di creazione e trasmissione della conoscenza e dell'innovazione. Una delle determinanti del successo dei distretti è rappresentata dalla capacità innovativa delle imprese, intesa non tanto come capacità di introdurre innovazioni radicali, mediante investimenti in ricerca e sviluppo, quanto piuttosto come capacità di migliorare i propri prodotti e/o processi e sviluppare la propria tradizione manifatturiera, grazie alla conoscenza del mercato di riferimento, alla padronanza di un materiale o di una tecnica produttiva, alla velocità di circolazione delle informazioni, al contatto interpersonale e all'osservazione diretta, generando processi di apprendimento on the job da parte della manodopera.

Ciò che favorisce questo coordinamento è la specifica struttura sociale ed economico-produttiva del territorio, che influenza le relazioni tra gli attori: individui, famiglie, imprese, istituzioni. La peculiare divisione sociale del lavoro è regolata da meccanismi di coesione e scambio sociale, nonché da codici comuni di comportamento, che sviluppano meccanismi di apprendimento collettivo e di riproduzione della conoscenza e riducono l'incertezza in cui si svolge l'attività economica. In tal modo, si ridimensionano i costi delle informazioni legati alle transazioni e si favoriscono la nascita e la localizzazione di nuove imprese.

Tale modello di organizzazione territoriale della produzione costituisce un concreto esempio dell'importanza delle relazioni di reciprocità e della strutturazione di un clima di fiducia per supportare l'intensificazione delle relazioni economiche su scala locale. Il fitto tessuto relazionale distrettuale, difatti, conferisce alle aziende rilevanti vantaggi competitivi embedded, incorporati nel territorio e fruibili solo dai soggetti operanti nello spazio locale. L'atmosfera industriale, creata dal distretto, determina l'accumulazione di complessi saperi a carattere tecnologico e gestionale su scala locale. Tale stock di know-how costituisce un patrimonio comune dal quale ciascuna impresa può attingere, attraverso le dense reti informative e relazionali, per incorporare le risorse di cui necessita nei suoi processi produttivi (Russo, 2012, p. 197).

Questi vantaggi hanno prodotto nel corso del tempo il cosiddetto "effetto distretto", che si traduce nel conseguire performance superiori da parte delle aziende localizzate nel distretto rispetto a quelle dello stesso settore ma localizzate fuori distretto. 
In una recente indagine ${ }^{6}$ si è inteso verificare l'impatto dei vantaggi competitivi delle imprese distrettuali del settore meccanico rispetto alle imprese dello stesso settore ma localizzate in aree non distrettuali. Considerato l'obiettivo dell'analisi, sono stati analizzati e messi a confronto per il periodo 2003-2007 i dati di bilancio di un campione di 1.769 imprese di 10 distretti del settore meccanico ${ }^{7}$ con quelli di 10.023 imprese meccaniche operanti su aree non distrettuali ${ }^{8}$.

In definitiva, si può affermare che per le imprese dei 10 distretti della meccanica osservati si rilevano perfomance di bilancio sostanzialmente differenti (in positivo), rispetto a quelle fatte registrare da imprese dello stesso settore ma non localizzate in distretti ${ }^{9}$.

I benefici dell'appartenenza ad un distretto sono maggiori per le imprese di piccole dimensioni ed in particolare per quelle inserite in distretti storici. I fattori che

6 La ricerca è stata coordinata dall'autore dell'articolo ed è stata realizzata da UniCredit Corporate Banking Progetti Speciali in collaborazione con Federazione dei Distretti Italiani. Per una sintesi della Ricerca (Ricciardi, 2011).

7 I dieci distretti esaminati sono: 1) Distretto della Meccatronica Pugliese - MEDIS (Bari); 2) Distretto Metalmeccanico Lecchese; 3) Distretto del Metallo delle Valli Bresciane (Lumezzane); 4) Distretto industriale del Coltello e della Metallurgia (Maniago); 5) Distretto Veneto del Condizionamento e della Refrigerazione Industriale (Padova); 6) Distretto produttivo della Meccatronica (Palermo); 7) Distretto industriale della Componentistica e Termoelettromeccanica CO.MET (Pordenone); 8) Distretto produttivo della Meccanica Siciliana (Siracusa); 9) Distretto della Termomeccanica - VenetoClima (Verona); 10) Metadistretto della Meccatronica e delle Tecnologie Meccaniche Innovative (Vicenza).

8 Per ciascuno dei 10 distretti, sono stati analizzati i dati di bilancio di un campione di imprese registrate in forma di società di capitali (S.p.A., S.r.l.).

Complessivamente sono stati esaminati i valori di bilancio degli ultimi 5 esercizi fiscali disponibili per un totale di 1.769 imprese. In particolare, sono stati calcolati i principali indici di performance (crescita del fatturato, margini operativi lordi), efficienza (ROI; Ebit/dipendenti) e sostenibilità finanziaria (D/E; PFN/Ebitda). Successivamente, i risultati raggiunti dal campione di imprese distrettuali sono stati confrontati con i risultati raggiunti da imprese non distrettuali. A tal fine, è stato costruito un benchmark rappresentativo di tutti i settori manifatturieri di specializzazione dei 10 distretti, costituito da 10.023 imprese meccaniche registrate sul territorio nazionale.

9 L'Ebitda (il Margine Operativo Lordo) in percentuale sul fatturato presenta per le aziende di distretto valori costantemente superiori a quelli registrati dalle imprese non distrettuali: il vantaggio è massimo $(+33 \%)$ per le imprese di dimensioni più piccole (fino a $15 \mathrm{mln} €$ di fatturato), si riduce per le imprese di medie dimensioni con fatturato fino a un massimo di $100 \mathrm{mln} €(+8 \%)$, mentre ritorna a crescere per le imprese di più grandi dimensioni con fatturato tra 200 e $500 \mathrm{mln} €(+25 \%)$. Inoltre, le imprese appartenenti ai distretti industriali presentano livelli di redditività del capitale investito (ROI) sensibilmente superiori in tutte le classi dimensionali. A beneficiare in misura maggiore dell'appartenenza al distretto sono le imprese piccole e piccolissime, che fanno registrare livelli medi di ROI superiori del $75 \%$ rispetto alle imprese operanti nello stesso settore, ma isolatamente. Il differenziale si riduce per le medie imprese $(+15 \%)$ per tornare a crescere nel caso delle imprese più grandi $(+45 \%)$. 
determinano questi benefici sono molteplici, tuttavia, quello determinante è l'opportunità di accesso a reti consolidate, che favorendo la cooperazione a diversi livelli (fornitori, sub-fornitori, imprese leader) permette, da un lato, alle imprese di grandi dimensioni di esternalizzare efficacemente parte della produzione (outsourcing) e, quindi, di generare livelli eccedenti di produttività, dall'altro lato, alle imprese di piccole dimensioni di accedere più agevolmente ad economie di scala, ottenendo vantaggi di perfomance chiaramente misurabili.

Un ulteriore effetto del vantaggio competitivo dei distretti è la diversa valutazione del rischio di impresa e quindi la conseguente determinazione dei tassi di interesse bancari nei differenti territori. Al riguardo, si rileva che nelle regioni dove non sono localizzati distretti i tassi di interesse sui finanziamenti sono più alti. In particolare, se si sovrappone la mappatura dei distretti industriali italiani rilevati dall'Istat in base al censimento del 2001 con quella dei tassi di interesse provinciali rilevati nel 2005 e nel 2009 si osserva che nelle province a maggiore vocazione distrettuale i tassi di interesse sono più bassi (Figg. 4, 5, 6).

Fig. 4: I 156 distretti individuati dall'lstat

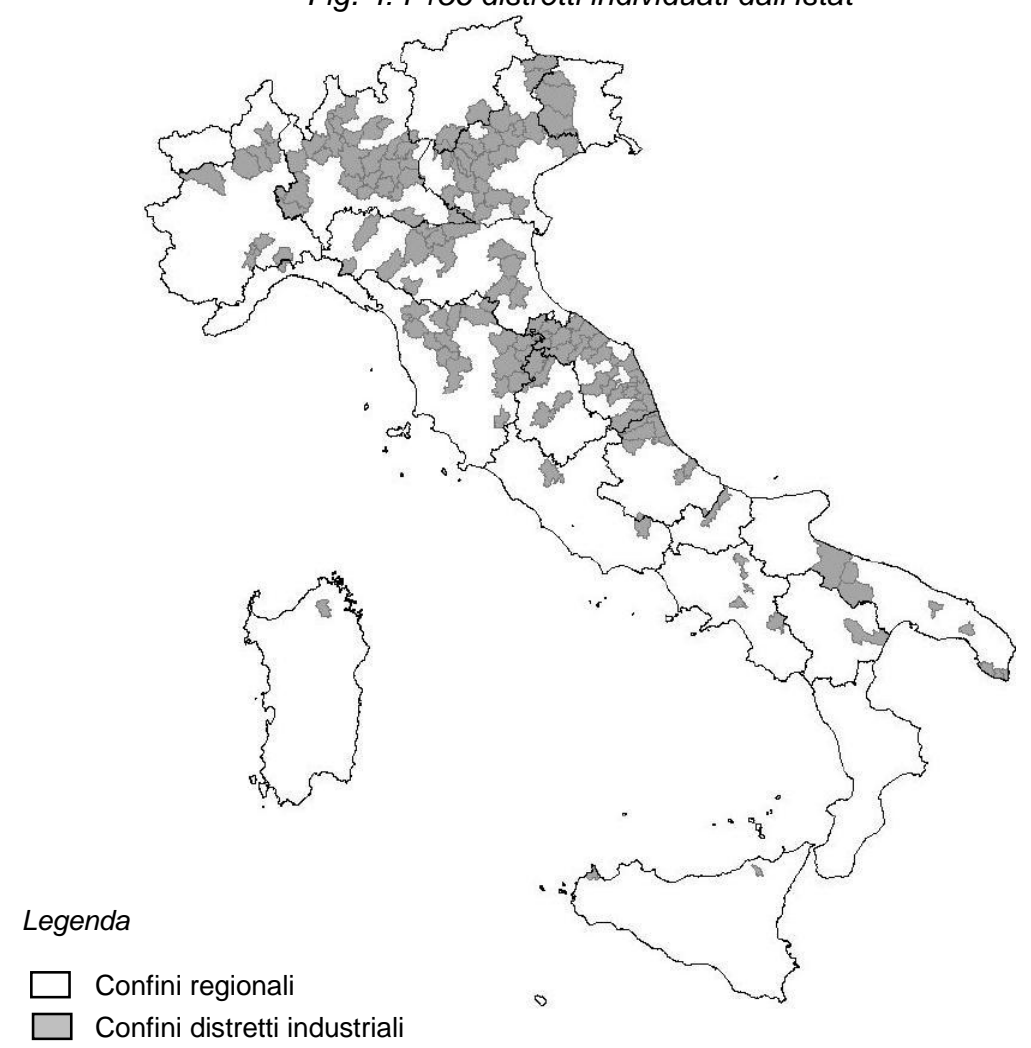

Fonte: Istat, 2005 
Fig. 5: Distribuzione dei tassi di interesse (2005)

Tassi di interesse sui prestiti a breve

4,00\% - 5,99\%

$6,00 \%-6,99 \%$

$7,00 \%-7,99 \%$

$8,00 \%-9,09 \%$

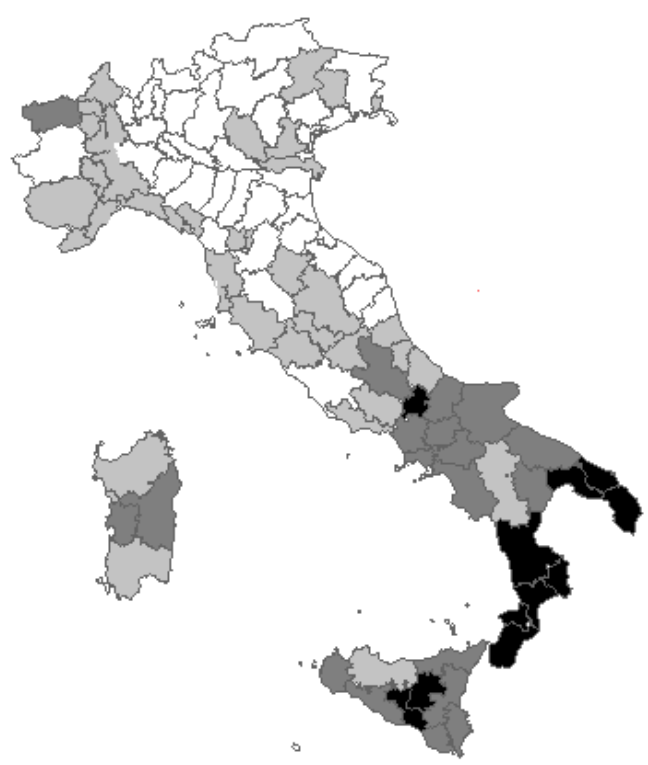

Fonte: ns. elaborazione su dati Unioncamere, 2006

Fig. 6: Tassi di interesse sui prestiti a breve termine (settembre 2009)

Tassi di interesse sui prestiti a breve

$3,5 \%-5 \%$

$\square 5 \%-6,5 \%$

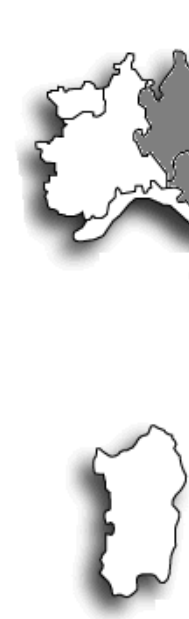

Fonte: ns. elaborazione su dati Banca d'Italia, 2009 


\section{Il ciclo di vita dei distretti}

Generalmente, il modello organizzativo del distretto presenta un processo evolutivo caratterizzato da tre fasi (v. Fig. 7): la prima di specializzazione della produzione, la seconda di irrobustimento dei rapporti tra le imprese, la terza di maturità.

Fig. 7: Il ciclo di vita dei distretti

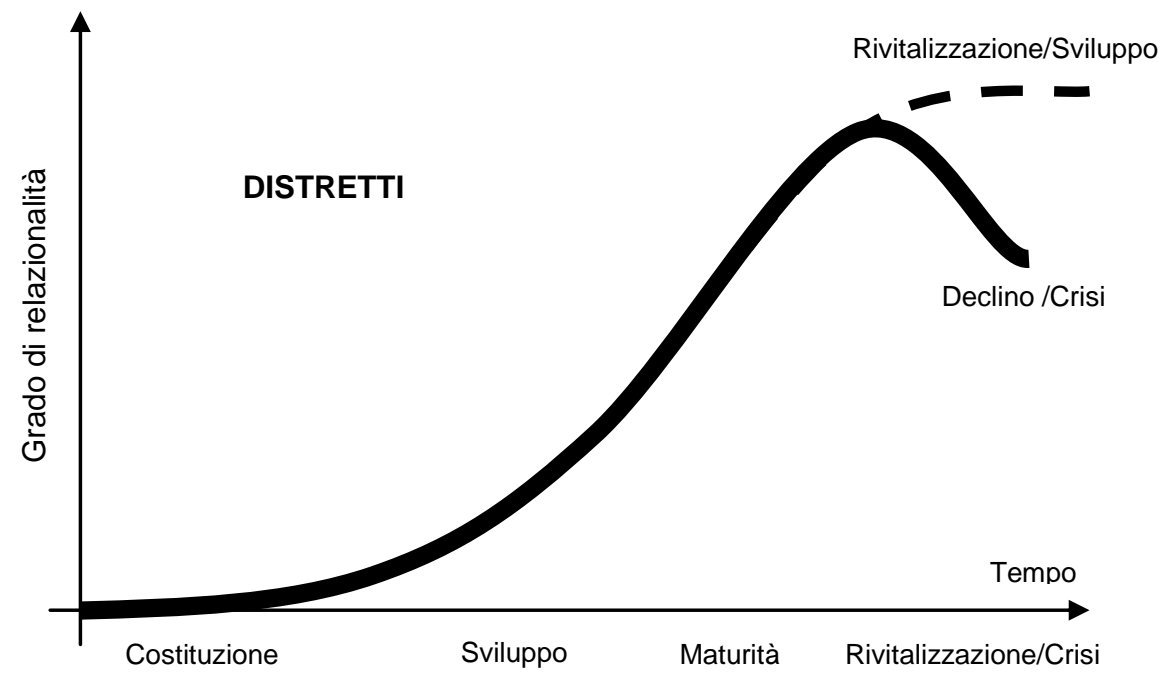

Fonte: ns. elaborazione

In questi ultimi anni si sta assistendo allo sviluppo della terza fase del ciclo di vita dei distretti, stimolato da una serie di criticità, sia a livello di contesto che di singola impresa. In particolare, quello che si osserva è che in un contesto competitivo fortemente turbolento il distretto non permette di fornire risposte soddisfacenti giacché le imprese non dispongono di conoscenze e competenze tali da poter fronteggiare le nuove dimensioni del processo concorrenziale. Si fa riferimento, in primo luogo, ai ritardi della tecnologia, alla debolezza delle politiche di marketing, in particolare all'impossibilità di attivare un controllo efficace sulla qualità e sui servizi erogati alla clientela finale, alla scarsa attenzione alla gestione finanziaria, al basso livello di pianificazione della cooperazione tra imprese.

In particolare, la struttura distrettuale manifesta considerevoli punti di debolezza soprattutto nelle funzioni in cui è determinante la dimensione organizzativa, come ad esempio, nell'applicazione delle innovazioni. In questo caso, infatti, la piccola dimensione delle imprese distrettuali non permette di fronteggiare il fabbisogno finanziario degli investimenti e il rischio connesso all'incertezza associata al lungo differimento temporale del recupero degli esborsi finanziari. Se a questi limiti si aggiungono alcuni fenomeni congiunturali, come ad esempio la concorrenza di 
alcune produzioni asiatiche e dell'est europeo che hanno beneficiato del trasferimento di know-how da parte delle stesse imprese di alcuni distretti ${ }^{10}$, alcuni problemi cronici di questo sistema, come le difficoltà connesse al ricambio generazionale degli imprenditori e degli stessi lavoratori, la carenza di professionalità specializzate, alcune scelte strategiche controproducenti come la delocalizzazione in aree dove non è possibile beneficiare del contesto socioambientale di riferimento ${ }^{11} \mathrm{e}$, non ultimo per importanza, l'immobilismo da parte degli enti locali che governano i distretti, si delinea un quadro prospettico piuttosto critico con ripercussioni sulla stessa sopravvivenza dei distretti nel medio-lungo periodo.

Attualmente, alcuni distretti stanno affrontando situazioni di crisi mentre altri stanno adottando strategie di rivitalizzazione che hanno contribuito, dopo un periodo di stasi congiunturale, ad un loro ulteriore sviluppo.

Quello che emerge dai dati export è che la crisi o il successo dei distretti non dipendono né dalla localizzazione geografica né dai settori di appartenenza (v. Figg. 8 e 9), ma dalle scelte strategiche messe in campo ${ }^{12}$.

10 È opportuno precisare al riguardo che la produzione dei distretti è minacciata anche dalla concorrenza sleale, soprattutto delle aziende cinesi, mediante una sistematica falsificazione dei prodotti e dei marchi delle aziende italiane. Gli effetti della contraffazione operata dalle aziende asiatiche sono rilevanti nei settori moda e arredo casa. A favorire il fenomeno è l'attività di grossisti e installatori che molto spesso in maniera consapevole favoriscono la penetrazione nel mercato dei falsi. Cfr. Fortis e Quadrio Curzio, 2003.

11 Sotto questo profilo, emblematico è il caso Said, azienda leader di utensili per la lucidatura di piastrelle e ceramiche. Per dieci anni, la Said ha fornito materie prime ai partner cinesi per realizzare il semilavorato, per poi completare il prodotto in Italia anche al fine di controllarne la qualità. Nel corso del tempo la delocalizzazione ha comportato numerosi problemi: eccessivi costi di trasporto e complessità della logistica, ma soprattutto il mancato rispetto della dichiarazione dei materiali da parte dei fornitori cinesi. Su questi presupposti la Said ha ritrasferito tutta la produzione a Isola Vicentina integrando verticalmente altre aziende del territorio creando un polo distrettuale con prodotti rigorosamente Made in Italy.

12 Nel settore del tessile-abbigliamento, ad esempio, nel 2010 la distanza tra i distretti migliori e quelli peggiori si è notevolmente ampliata: il distretto di Biella, specializzato in produzioni di alta qualità, ha registrato una crescita superiore al $20 \%$ contro un calo sensibile del fatturato registrato nel distretto di Corato (Puglia) e in quello delle confezioni e abbigliamento della Bassa bresciana. Meno sensibile ma altrettanto significativa è la differenza tra i distretti nel settore del legno-arredo: da un lato, si rileva la crescita del $10 \%$ del fatturato nel distretto del mobile di Forlì e del 5\% in quello della provincia di Pordenone, dall'altro lato una riduzione di circa 1' $8 \%$ in quello del mobile classico della pianura veneta. Nel settore legno-arredo i buoni livelli di redditività di alcuni distretti sono stati raggiunti anche grazie a progetti di ricerca condotti in collaborazione con centri universitari, alle certificazioni di qualità, al potenziamento della fase commerciale e alla diversificazione produttiva. 
Fig. 8: Andamento delle esportazioni dei distretti di "successo" (var. \% su valori nominale 2010/2011)

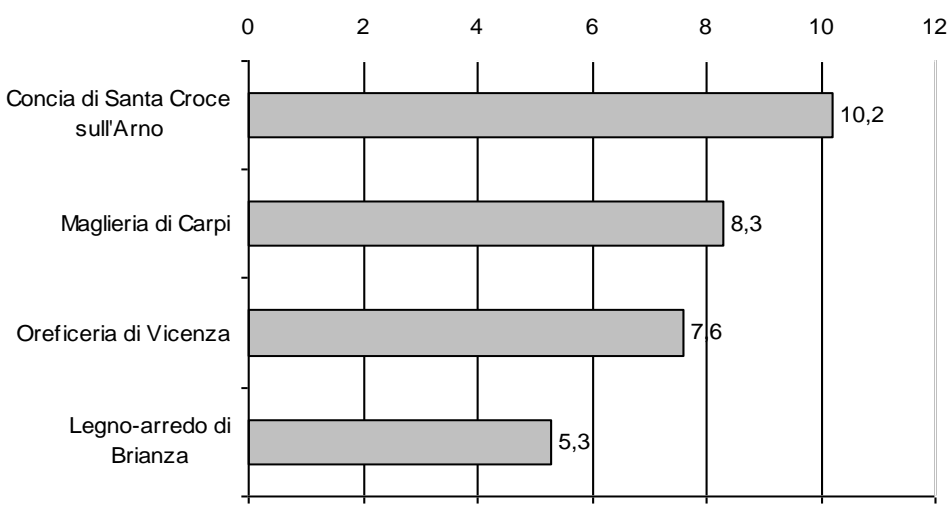

Fonte: Intesa Sanpaolo, Monitor dei Distretti, aprile 2012

Fig. 9: Andamento delle esportazioni dei distretti in crisi (var. \% su valori nominale 2010/2011)

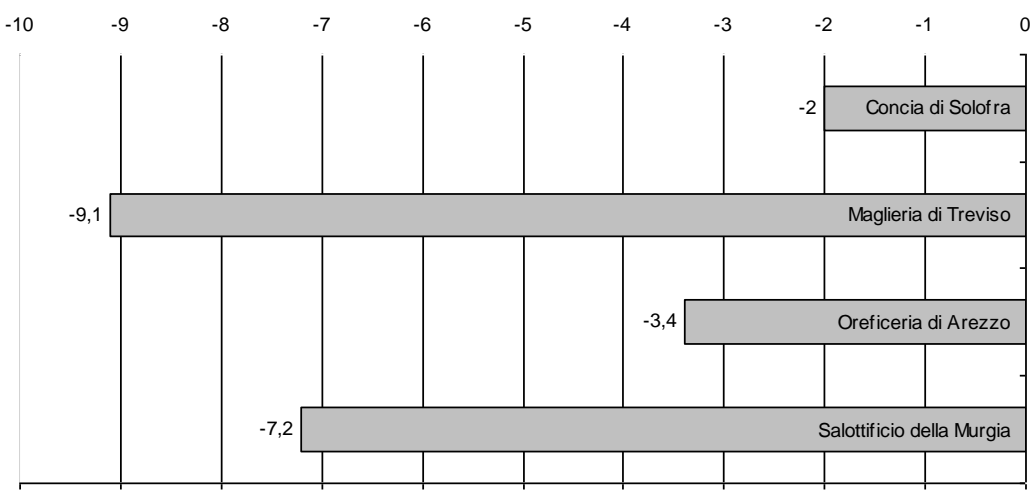

Fonte: Intesa Sanpaolo, Monitor dei Distretti, aprile 2012

Le principali cause che hanno determinato la crisi di alcuni distretti sono ${ }^{13}$ :

13 Esempio tipico di distretto in crisi è quello del salotto della Murgia che negli ultimi anni ha registrato un sensibile calo di ordini, chiusura di aziende e conseguenti licenziamenti. Queste performance negative sono state determinate da una serie di inefficienze. In primo luogo, le imprese del distretto hanno manifestato un'eccessiva dipendenza dalla più importante impresa leader (Gruppo Natuzzi) che ha provocato la mancanza di stimoli ad applicare innovazioni di prodotto e di processo e la cui crisi ha determinato a cascata la 
- incapacità a controllare i mercati di sbocco e scarsa attenzione ai mutamenti della domanda;

- bassi livelli di investimenti in innovazione di prodotto e di processo;

- limitate competenze nella gestione finanziaria;

- carenza di professionalità specializzate;

- scarsa cooperazione tra le imprese;

- concorrenza, qualche volta anche sleale, di alcune produzioni asiatiche e dell'est europeo;

- limitato sostegno da parte degli enti locali.

Esistono, tuttavia, distretti in forte sviluppo che hanno saputo riconquistare livelli di competitività grazie all'implementazione di strategie vincenti ${ }^{14}$ :

- cooperazione con altre imprese fino ad arrivare alla costituzione di reti di imprese;

- sforzo congiunto nell'applicazione di innovazioni di prodotto e di processo;

- strategie comuni di marketing e formazione del personale;

- delocalizzazione pianificata;

- presenza di numerose aziende leader che coordinano differenti filiere;

- elevata autonomia delle imprese sub-fornitrici;

- sinergie con università e centri di ricerca;

- collaborazione con le parti sociali e governance efficiente da parte degli enti locali.

\section{La nuova classificazione dei distretti}

La presenza di distretti con performance diverse non dipende dai settori di appartenenza e/o dalla loro localizzazione, in quanto all'interno degli stessi settori e in aree limitrofe coesistono distretti in crescita e distretti in difficoltà ${ }^{15}$. Inoltre, si rileva che negli stessi distretti in crescita operano aziende in crisi e nei distretti in crisi operano aziende in forte sviluppo. In definitiva, il successo o la crisi dei

crisi delle altre imprese. Si rileva, poi, una fortissima incidenza di microimprese (il 50\% occupa da 1 a 15 dipendenti) che hanno mostrato scarsa attenzione ai mutamenti della domanda e all'innovazione del prodotto.

14 Esempio di distretto di successo è quello metalmeccanico di Lecco (1.089 imprese, 21.000 addetti, 2 miliardi di euro di fatturato, il $40 \%$ export ) che ha registrato negli ultimi anni un sensibile incremento delle esportazioni grazie a:

- notevoli investimenti in innovazione (il $41,3 \%$ delle imprese investe in innovazione dal 6 al 10\% del fatturato);

- rapporti collaborativi con le parti sociali;

- presenza di reti di imprese che condividono investimenti in marketing, formazione, ricerca e sviluppo;

- rafforzamento del patrimonio delle aziende che ha permesso un aumento delle loro dimensioni mediante capitalizzazione.

15 Per ulteriori approfondimenti si veda il paragrafo 4 di questo stesso articolo. 
distretti e delle loro aziende dipende dalla qualità della governance e dalle strategie adottate. Sotto questo profilo, i distretti che mostrano performance migliori sono caratterizzati da alcuni fenomeni comuni: propensione all'investimento in innovazione; governance efficiente; presenza di diverse aziende leader che coordinano numerose filiere; elevata autonomia delle imprese subfornitrici; sinergie con università e centri di ricerca ${ }^{16}$.

In base all'evoluzione che caratterizza i singoli distretti, in funzione della loro governance e delle strategie implementate è stata elaborata una nuova classificazione dei distretti (Ricciardi, 2010-a; Fazio e Ricciardi, 2008):

a. distretti dinamici: dotati di governance efficiente, mettono in campo strategie che garantiscono lo sviluppo e l'incremento costante delle performance reddituali e delle quote export. Ciò che contraddistingue questi distretti è l'intensa cooperazione tra le imprese con una forte interdipendenza dei loro cicli produttivi che favorisce la presenza di un circuito di conoscenza; le imprese leader, non sempre quelle di maggiori dimensioni, si propongono come laboratori di innovazioni strategiche e condizionano "virtuosamente" i comportamenti di tutte le altre aziende di distretto;

b. distretti maturi: presentano un forte radicamento al territorio d'origine e alla comunità locale ma sono privi di una governance efficiente e la cooperazione tra le imprese è di natura spontanea e non pianificata. Inoltre, le imprese sono poco attente all'innovazione di prodotto e al marketing. Subiscono pesantemente le crisi congiunturali ma in alcuni casi, grazie alle loro capacità imprenditoriali, riescono in parte a superarle;

c. distretti vulnerabili: privi di governance e con un debole radicamento sul territorio. Le imprese non sviluppano strategie di cooperazione, operano isolatamente e molto spesso in concorrenza tra loro; l'appartenenza al distretto, in presenza di crisi congiunturali, costituisce un fattore di rischio piuttosto che un'opportunità, soprattutto quando si rileva la presenza di una o poche imprese leader che rappresentano l'unico sbocco commerciale. Inoltre, l'assenza di investimenti in innovazione e di strategie di commercializzazione contribuisce a rendere fortemente instabili le performance di questa categoria di distretti;

d. distretti virtuali: esistono sulla carta, «imposti» per decreti regionali, ma di fatto rappresentano solo aree industriali.

16 Il distretto Veneto del Condizionamento e della Refrigerazione Industriale di Padova, ad esempio, registra le migliori performance tra i distretti della meccanica grazie a una governance efficiente, garantita dalla presenza di Refricold Scarl che svolge funzioni di coordinamento e che promuove iniziative e progetti comuni. Inoltre, il Distretto si caratterizza per gli intensi rapporti di collaborazione sia tra le imprese sia con l'Università di Padova e per la presenza di imprese, di medie e piccole dimensioni, con forte propensione all'investimento in innovazione, capacità di attrarre figure manageriali esterne e dotate di elevata autonomia rispetto alle imprese leader anche mediante la diversificazione della clientela localizzata al di fuori del distretto. Queste imprese, tra l'altro, stanno avviando la transizione dalla governance familiare ad una piena delega manageriale (UniCredit Corporate Banking e Federazione dei Distretti Italiani, 2009). 
Fig. 10: La nuova classificazione dei distretti italiani: i distretti dinamici

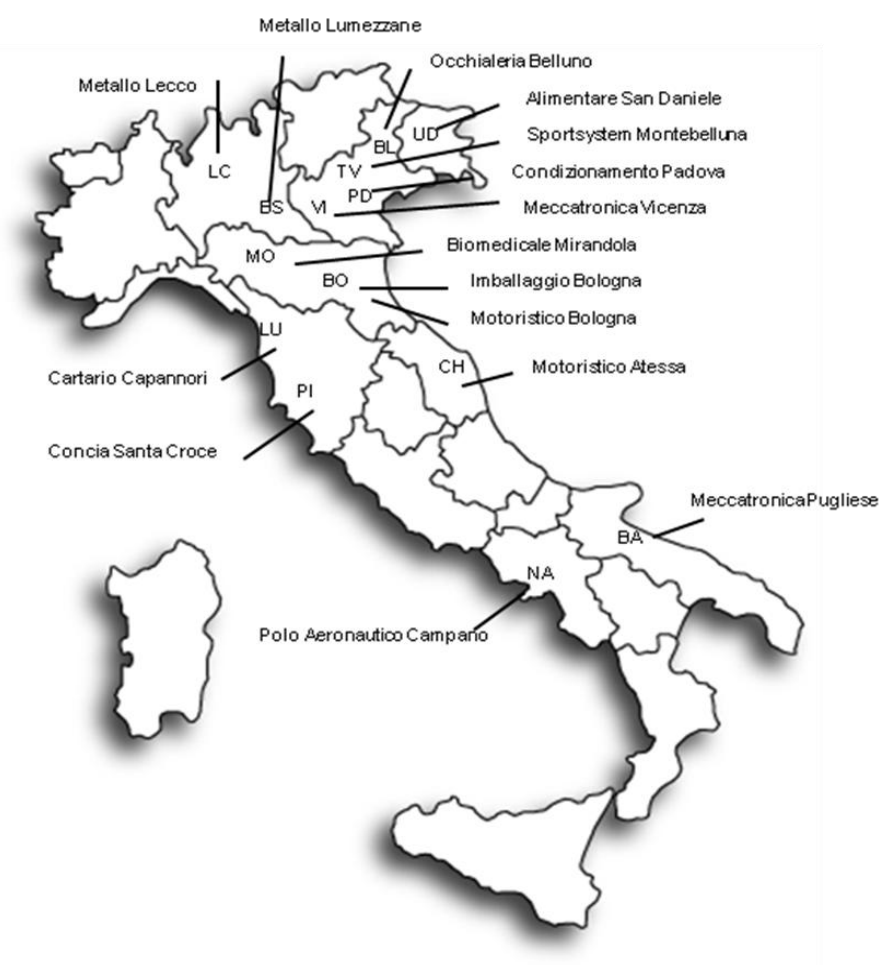

Fonte: ns. elaborazione

È interessante rilevare che uno degli elementi sempre presente in tutti i distretti dinamici è la governance efficiente ${ }^{17}$. Una recente ricerca (Pastore e Tommaso, 2012) ha dimostrato empiricamente una correlazione tra performance economicofinanziarie e qualità della governance dei distretti. L'indagine ha riguardato 10 distretti: 5 distretti con performance superiore alla media di settore e 5 distretti con

17 A Mirandola (Modena), il Consorzio ConsoBioMed, ente di governance del distretto nato nel 1990, ha contribuito a favorire la cooperazione tra le imprese ed è diventato un punto di riferimento per l'applicazione delle innovazioni e per l'internazionalizzazione. Grazie alle iniziative del Consorzio il distretto è conosciuto ed apprezzato in tutto il mondo per le sue macchine elettromedicali e per la produzione di organi artificiali.

Nel distretto cartario di Capannori (Lucca) è stato costituito Lucense, un centro multifunzionale capace di proporre soluzioni ai problemi tecnologici e ambientali delle singole aziende. In particolare, è stato realizzato un prototipo di impianto in grado di rendere efficiente il ciclo degli scarti in linea con quanto già previsto da una Direttiva europea che imporrà ai rifiuti industriali delle carte da macero di produrre energia. 
performance inferiore alla media di settore ${ }^{18}$. In particolare, nei distretti industriali caratterizzati dalla presenza di strutture di governance che hanno intrapreso azioni efficaci sul territorio e dotati di strumenti efficienti di coordinamento, le imprese ottengono risultati economici e finanziari mediamente superiori; viceversa, nei distretti caratterizzati da assenza di governance oppure sistemi di governance inefficienti in termini di iniziative e strategie, le performance economico-finanziarie delle aziende sono inferiori alla media.

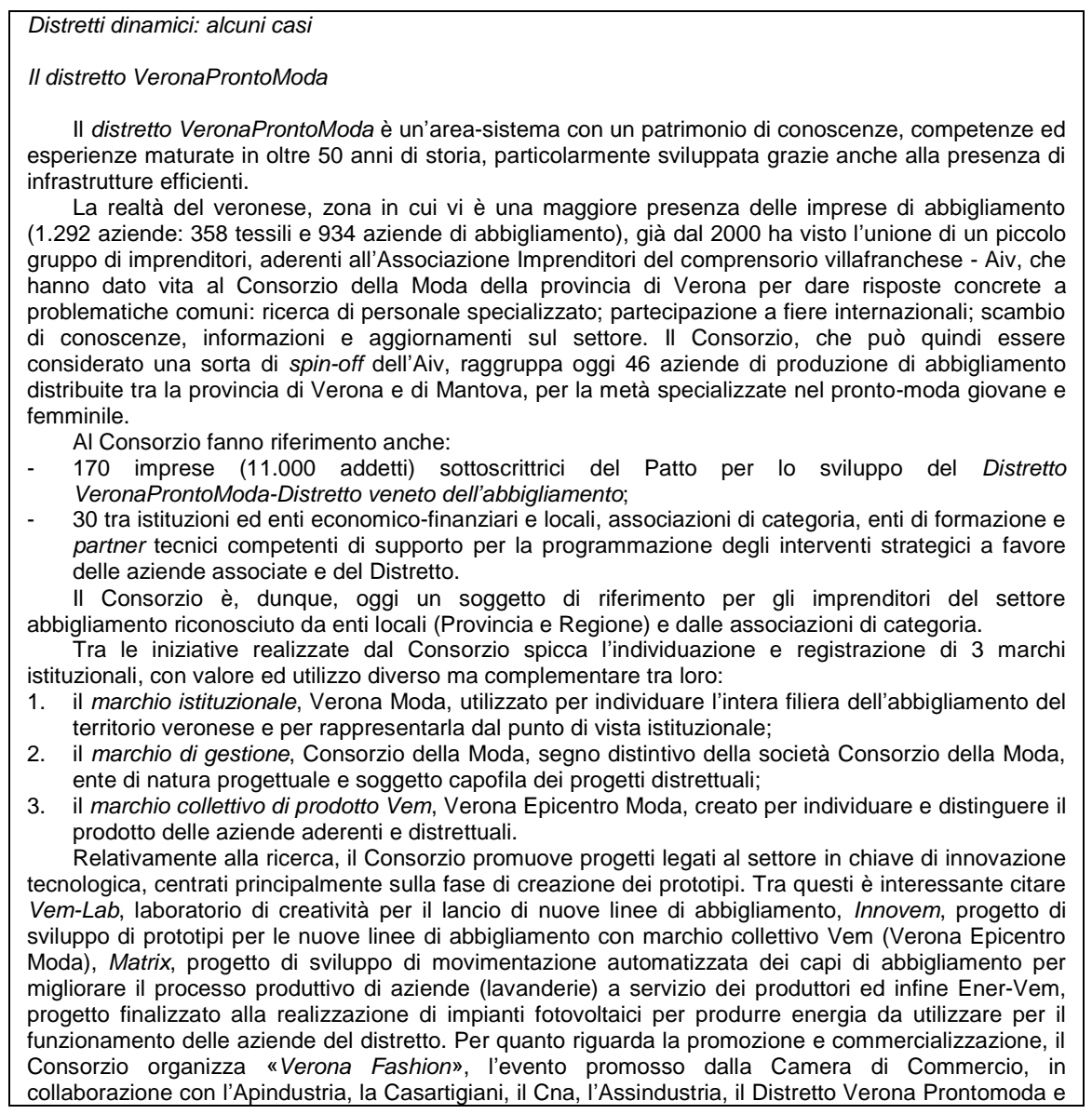

18 I dieci distretti dell'indagine appartengono ai settori meccanico, abbigliamento-moda e arredo-casa. La qualità della governance è stata valutata in funzione della presenza di strutture di governance; presenza di strumenti di governance; efficacia delle azioni intraprese. Quali indicatori di performance economico-finanziarie sono stati considerati: la variazione percentuale del fatturato e dell'export; il Roi, il Roe e il grado di patrimonializzazione medio. 
I'Unione Provinciale Artigiani, per dare visibilità e valorizzazione alle imprese veronesi del settore tessile-abbigliamento.

Una caratteristica comune alle aziende del consorzio è quella di aver realizzato un intenso processo di outsourcing delle fasi produttive a maggior intensità di manodopera anche se, evidentemente, non tutte le aziende presentano la stessa situazione. In particolare, il confezionamento dei capi avviene prevalentemente attraverso l'ausilio di laboratori che lavorano in conto terzi: il $90 \%$ delle aziende di abbigliamento affida a terzisti (per lo più laboratori che generalmente fanno parte del Distretto VeronaProntoModa) la fase della confezione. Le aziende presidiano direttamente e governano al proprio interno le attività legate allo styling, alla modellistica, alla commercializzazione e alla logistica. La maggior parte delle aziende outsourcer sono fornitori stabili e affidabili interni al Distretto sebbene ci siano anche molti laboratori cinesi, non inseriti nel distretto. La rete di fornitura è soprattutto locale: il $60 / 70 \%$ delle aziende fornitrici è collocata entro i $100 \mathrm{~km}$, il resto è rappresentato da aziende localizzate sul territorio nazionale anche perché la stessa natura dei cicli produttivi estremamente rapidi non rende praticabile la via della delocalizzazione. Le aree storiche della sub-fornitura del pronto moda veronese si collocano in Emilia-Romagna, precisamente nella zona di Carpi, in Lombardia e, in misura minore, in Toscana. Inoltre, il Consorzio ha avviato una collaborazione strategica tra aziende dell'area Verona/Veneto e aziende in Campania, in Toscana e in Puglia.

L'obiettivo perseguito è anche quello di completare la filiera mantenendo tutte le fasi di processo in Italia e quindi incentivando il vero Made in Italy.

Cfr. Ricciardi e Pastore, 2010; Ricciardi, 2009.

\section{Il distretto aeronautico campano}

In controtendenza rispetto all'andamento negativo dell'economia regionale, la fase di sviluppo e di crescita dell'attività aeronautica campana è trainata da Alenia Aeronautica, leader in Italia e tra le prime nel mondo per la produzione di aerei civili e militari, che racchiude conoscenze, tecnologie e strumentazioni all'avanguardia. In tale ambito, Alenia Aeronautica coordina una rete di Pmi-fornitori di secondo livello, altamente specializzate nella produzione di parti, componenti o interi gruppi funzionali per il settore aeronautico e spaziale e un insieme cospicuo di piccole imprese-subfornitori, che dispongono di tecnologie e processi produttivi compatibili con gli standard tecnici richiesti dal settore.

Alenia Aeronautica ha implementato un sistema di relazioni di outsourcing fondato prevalentemente su meccanismi di governo di natura gerarchico-cooperativa. Ciò vuol dire che nell'ambito del sistema di fornitura, Alenia si afferma quale vertice strategico-decisionale, le cui risorse e competenze chiave sono focalizzate sulle attività a maggior valore aggiunto (ricerca tecnologica $e$ assemblaggio finale) mentre definisce legami di partnership forti, non necessariamente equity, con imprese specializzate, di elevate competenze tecnico-organizzative e affini in termini di vision e cultura aziendale. Inoltre, la prossimità geografica rappresenta un ulteriore fattore strategico per Alenia: circa il $75 \%$ dei suoi sub-fornitori è localizzato a Pomigliano d'Arco nei pressi dello stabilimento principale, in cui si svolge l'assemblaggio finale.

II ridotto numero dei fornitori, opportunamente selezionati per qualificazione tecnica e tecnologica, ha aumentato nel tempo l'intensità nelle relazioni e la durata dei rapporti. La tendenza a stabilire accordi di lungo periodo migliora l'intesa fra le imprese e l'integrazione delle attività consente al fornitore una migliore pianificazione della produzione, oltre ad assicurargli il recupero dei costi relativi agli investimenti. L'idea di fondo è che la divisione del lavoro tra imprese, da un lato, favorisce il trasferimento di conoscenza su scala internazionale, non solo incorporandola nei prodotti ma anche sviluppando rapporti di cooperazione interaziendale e, dall'altro lato, esplica effetti moltiplicativi anche sulle attività delle imprese locali, in termini di spillover tecnologici e di mercato, trasferimento del sapere tecnologico, diffusione di processi di spin-off mettendo a disposizione un patrimonio di conoscenze altrimenti inaccessibile.

Una caratteristica importante da sottolineare è che nel settore aeronautico le aziende leader sempre più spesso richiedono l'assunzione di rischio da parte dei loro fornitori. La partecipazione di Alenia al programma A380, ad esempio, prevede, su un costo totale di 12 miliardi di euro, un investimento di oltre un miliardo di euro. A loro volta i sub-fornitori di Alenia versano a quest'ultima una quota, assumendosi anch'essi una parte del rischio legato al programma. L'assunzione di rischio da parte delle varie aziende che partecipano al programma consente, da un lato, al prime di ridurre i propri rischi e l'impegno finanziario, dall'altro alle imprese fornitrici di essere, coerentemente con la quota di capitale impegnata, non meri esecutori di parti, ma veri e propri partner.

Attualmente è in corso un processo di emancipazione delle imprese minori dall'impresa centrale (Alenia Aeronautica). Le Pmi aeronautiche italiane che oggi svolgono attività di sub-fornitura non solo per Alenia, ma anche a livello internazionale sono, in alcuni casi, il risultato di processi di spin-off dalla 
stessa Alenia. Le imprese in grado di realizzare un prodotto finito a marchio proprio hanno puntato sulla specializzazione di nicchia, soprattutto verso il segmento degli aerei superleggeri e dei piccoli velivoli da 6-8 posti: è la scelta, per esempio, compiuta da Vulcanair e da Tecnam. Altre aziende hanno perseguito una strategia di accesso alle reti di eccellenza transnazionali, anche attraverso processi di acquisizione (come è accaduto per Aerosoft). Infine, per alleggerire il vincolo di dipendenza da Alenia, altre imprese invece hanno preferito una strategia di diversificazione, affacciandosi in segmenti industriali contigui per tecnologie e competenze, come i trasporti marittimi e ferroviari (è il caso di Geven).

A livello di settore, si sta progettando di dare vita a un «metadistretto» tra le 5 aree forti del nostro Paese"(Campania, Lombardia, Piemonte, Lazio, Puglia), capace di competere sui scala globale mettendo insieme i poli di eccellenza geograficamente lontani ma assolutamente vicini se si guarda alle sinergie tecnologiche e produttive maturate negli ultimi anni. In questa direzione, Puglia e Campania hanno mosso i primi passi aggregandosi tra loro. Le due regioni hanno, infatti, sottoscritto un protocollo d'intesa per la realizzazione di un «Distretto dell'industria aeronautica e aerospaziale del Mezzogiorno", fondato sulla presenza nelle due regioni delle più grandi imprese italiane del settore e di un sistema diffuso di Pmi che compongono la filiera della subfornitura. II distretto unico, che sarebbe composto da 150 imprese, per un fatturato complessivo di 2 miliardi di euro (pari al $32 \%$ del totale italiano), punta a sfruttare le potenzialità derivanti dal trend di forte crescita internazionale che caratterizza il settore aeronautico in questa fase congiunturale.

Cfr. Ricciardi, 2008-b.

\section{Le recenti tendenze evolutive: i risultati dell'Osservatorio Distretti Italiani}

L'Osservatorio Nazionale dei Distretti Italiani, istituito nel 2009, grazie al contributo di autorevoli partner ${ }^{19}$, rappresenta la banca-dati ufficiale dei distretti italiani e persegue gli obiettivi di aggiornare e monitorare periodicamente i dati relativi ai distretti e studiare i fenomeni che ne caratterizzano l'evoluzione e la trasformazione.

L'Osservatorio dei Distretti Italiani analizza 101 distretti $^{20}$, la maggior parte dei quali localizzati nel Nord Italia (Fig. 11) e così ripartiti per settori di specializzazione: Abbigliamento-Moda (37,6\%); Arredo-casa (21,8\%); Automazione e meccanica $(25,7 \%)$; Alimentare $(11,8 \%)$. Si veda, al riguardo, la Fig. 12 .

Nel 2010, nei 101 distretti dell'Osservatorio risultano registrate 283mila aziende (erano 285mila nel 2009) il 28,5\% del totale manifatturiero. Nel 2009, le imprese attive nei distretti impiegavano oltre 1,4 milioni di addetti (fonte Istat), che rappresentano il $29,4 \%$ del totale dell'occupazione manifatturiera.

19 Banca d'Italia, Censis, Cna, Confartigianato, Confindustria, Federazione dei Distretti Italiani, Fondazione Edison, Intesa Sanpaolo, Istat, Symbola e Unioncamere.

20 I 101 distretti del campione Osservatorio comprendono i distretti aderenti alla Federazione dei Distretti Italiani e quelli considerati nella $9^{\text {a }}$ Indagine annuale su «Le medie imprese industriali italiane» a cura di Unioncamere e Mediobanca. Per ulteriori informazioni sulla metodologia seguita si veda la documentazione disponibile sul Portale dell'Osservatorio http://www.osservatoriodistretti.org. 
Fig. 11: I distretti dell'Osservatorio per Regione

Fonte: ns. elaborazione

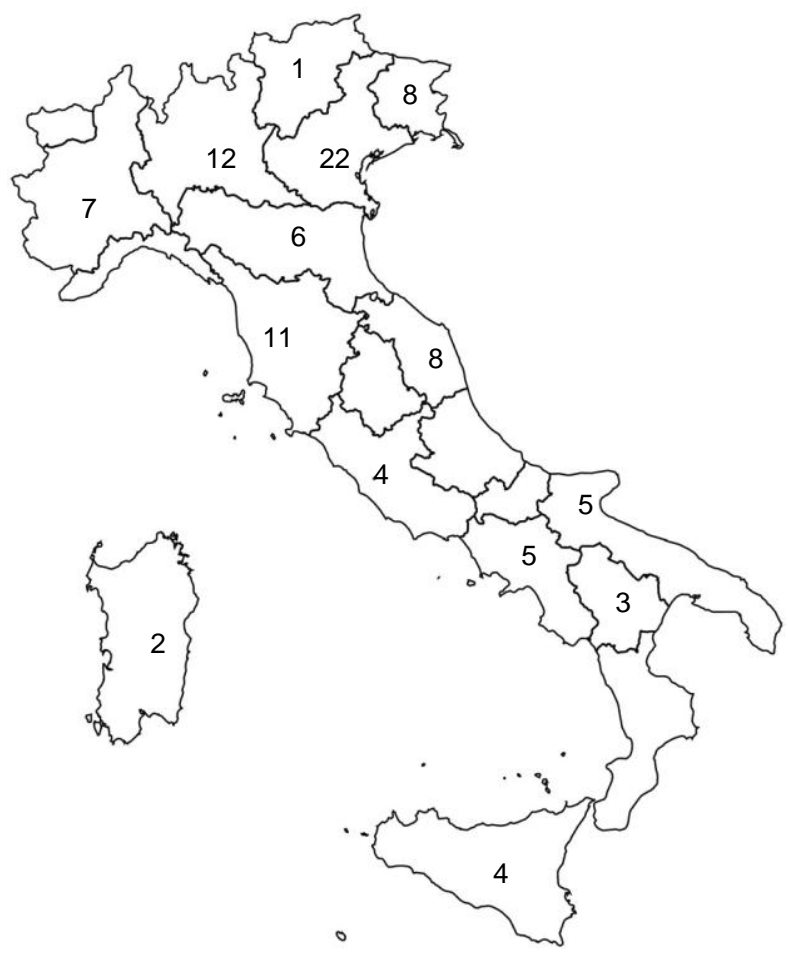

Fig. 12: I distretti dell'Osservatorio per settore di specializzazione

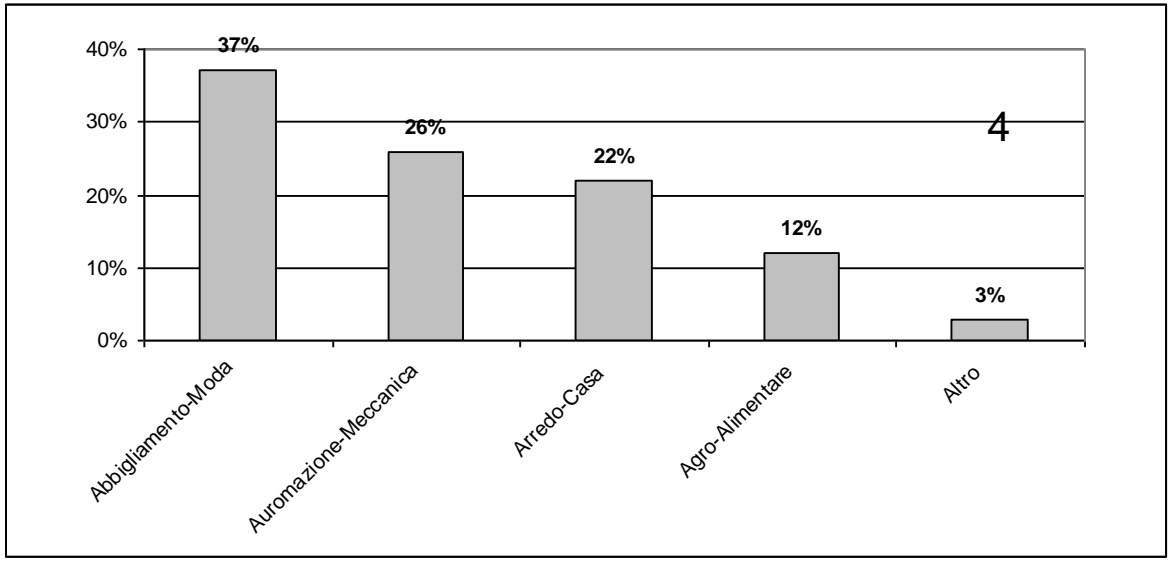

Fonte: ns. elaborazione 


\subsection{Le performance delle imprese dei distretti nel periodo $2008-2011^{21}$}

I risultati dei bilanci 2008-2009 delle imprese distrettuali hanno mostrato con molta evidenza gli effetti della crisi economica. In base alle Indagini elaborate dal Servizio Studi e Ricerche di Intesa Sanpaolo e pubblicate nei Rapporti dell'Osservatorio, nel 2008 il fatturato delle imprese distrettuali si è ridotto del 3,1\% ed ha accusato un ulteriore sensibile ridimensionamento nel 2009, arretrando in termini mediani del $18,9 \%$. I settori di specializzazione che hanno mostrato le maggiori difficoltà sono stati quelli metalmeccanico, sistema moda e sistema casa.

Solo le imprese dei distretti alimentari, grazie al carattere poco ciclico dei consumi, sono riuscite nel 2009 a limitare al minimo il calo delle vendite (nel 2008 questi distretti avevano registrato un aumento del fatturato del $5 \%$ ).

Dopo il crollo del biennio 2008/09, il fatturato delle imprese distrettuali ha registrato nel 2010 un aumento medio dell' $8,2 \%$ : le performance migliori sono state ottenute dai distretti della meccanica $(+13,4 \%)$ e dal sistema moda $(+9 \%)$ mentre più contenuta è la crescita nel settore agroalimentare $(+2 \%)$. Volendo fare un raffronto con il fatturato del 2008, anno pre-crisi, solo il settore alimentare ha superato quei livelli $(+2,1 \%)$ mentre tutti gli altri settori di specializzazione sono ancora molto lontani: metalmeccanica $(-17,3 \%)$, sistema casa $(-16,8 \%)$, sistema moda $(-8,8 \%)$. In linea generale il fatturato dei distretti nel 2010 è stato del 12,1\% inferiore a quello fatto registrare nel 2008.

Il crollo del fatturato ha influenzato pesantemente il valore dei due principali indici di redditività: Roi e Roe, rispettivamente pari al 5,1\% e 2,8\% nel 2010, pur avendo registrato un aumento rispetto al 2009 (4,3\% e 1,3\%), sono comunque ancora molto al di sotto dei livelli raggiunti nel $2008(6,9 \%$ e 3,5\%) (Tab. 1).

Tab. 1: Indici e dati di bilancio delle aziende distrettuali nel periodo 2008-2010

\begin{tabular}{|lccc|}
\hline & 2008 & 2009 & 2010 \\
\hline Fatturato & $-3,1 \%$ & $-18,9 \%$ & $+8,2 \%$ \\
\hline Roi & $6,9 \%$ & $4,3 \%$ & $5,1 \%$ \\
\hline Roe & $3,5 \%$ & $1,3 \%$ & $2,8 \%$ \\
\hline
\end{tabular}

Fonte: ns. elaborazione

Gli effetti della crisi si sono abbattuti soprattutto sulle microimprese che hanno registrato nel periodo 2008/2010 un calo del fatturato pari al 13\% e una riduzione del Roi pari al 2,1\% contro un ridimensionamento rispettivamente dell'11,4\% e

21 L'analisi di questa indagine è realizzata sui bilanci di esercizio del triennio 2008/10 estratti dal data base del servizio studi di Intesa Sanpaolo e relativi alle imprese dei distretti individuati dall'Osservatorio nazionale distretti italiani. Sulla base di questi criteri sono state individuate 14.399 imprese distrettuali con fatturato superiore ad un milione di euro. Per un approfondimento del campione si rimanda ai capitoli elaborati da Intesa Sanpaolo nei Rapporti dell'Osservatorio. 
dell'1,4\% delle medie imprese. Le previsioni reddituali per le aziende di minori dimensioni sono molto negative in quanto, rispetto alle altre aziende, presentano livelli di indebitamento molto più elevati (pari a circa il $70 \%$ delle fonti di finanziamento) che determineranno pesanti oneri finanziari nella prospettiva di sensibili rialzi dei tassi di interesse ${ }^{22}$.

Si conferma il fenomeno della dispersione delle performance: nel 2010, in termini di variazione del fatturato, il differenziale tra imprese migliori e imprese peggiori si è ulteriormente ampliato, per cui la mediana della crescita del fatturato delle imprese distrettuali migliori è stata pari al 48,3\% mentre quella delle imprese peggiori è stata pari a $-24,4 \%$ con uno scarto di quasi il $73 \%$ (era del $57 \%$ nel 2009). L'alta dispersione di performance riguarda sia aziende localizzate in differenti distretti ma appartenenti allo stesso settore di specializzazione sia aziende localizzate negli stessi distretti. Nel primo caso, la dispersione è determinata dal diverso posizionamento strategico dei distretti, in termini di innovazioni di prodotto, servizi alla clientela, canali distributivi; nel secondo caso dal diverso ruolo svolto dalle imprese all'interno della filiera produttiva. Sotto questo profilo, le imprese capofila (leader) e quelle fornitrici di "primo livello" (specializzate) hanno avuto la capacità di offrire prodotti esclusivi e personalizzati, che hanno garantito un'alta redditività e un soddisfacente portafoglio ordini; viceversa le microimprese, "fornitrici di capacità produttiva" sono state penalizzate dai minori ordini delle imprese capofila, che hanno internalizzato parte della produzione precedentemente esternalizzata. Quello che si rileva anche da recenti studi (Gallo, 2011, p. 163) è che le imprese che hanno perseguito strategie di leadership sono più redditizie e caratterizzate da un più elevato contenuto immateriale della produzione che si riflette in un marchio affermato e in una produzione di qualità. Sono soprattutto queste le imprese che, accompagnate da un'attenta e oculata gestione finanziaria, stanno ampliando il controllo delle fasi di gestione focalizzando risorse anche sulla distribuzione e vendita del prodotto soprattutto all'estero, ricercando nuove nicchie di mercato e nuove opportunità in aree di business complementari a quelle di specializzazione del distretto ${ }^{23}$. Imprese tanto evolute strategicamente che in alcuni

22 Sotto il profilo finanziario, ad aggravare la posizione delle micro e piccole imprese sono le condizioni di incasso dei crediti, le cui scadenze, dopo il balzo del 2009, sono ulteriormente aumentate, determinando un pericoloso circolo vizioso: peggioramento della qualità del capitale circolante con conseguente deterioramento del rating e contestuale necessità a ricorrere ad un massiccio indebitamento a breve a tassi molto onerosi.

23 Anche le ricerche condotte dalla Banca d'Italia (cfr. I distretti industriali nelle analisi sulle economie regionali della Banca d'Italia, www.osservatoriodistretti.org) evidenziano la crescente eterogeneità delle strategie e dei risultati delle imprese all'interno dei distretti. In particolare, nei distretti calzaturieri marchigiani, le performance migliori negli anni più recenti sono state ottenute da quelle imprese che hanno adottato una strategia di differenziazione qualitativa, supportata da investimenti nel marchio, nella ricerca e sviluppo, in reti commerciali dedicate. Nei distretti del Nord est le imprese più 
casi hanno sostenuto gli anelli deboli della filiera (deboli soprattutto per criticità finanziarie) al fine di garantire nel medio-lungo periodo la flessibilità produttiva e la «conservazione» nel territorio della qualità della produzione.

\subsection{I risultati del 2011}

In base all'indagine campionaria di Unioncamere (Cap. 2 del Terzo Rapporto), la quota di aziende distrettuali che ha segnalato un incremento del fatturato nel 2011 è aumentata rispetto al 2010, passando dal 34\% al 39,9\%, quota superiore a quella delle aziende manifatturiere non distrettuali $(37,2 \%)$. Un incremento del fatturato superiore alla media lo hanno registrato le aziende dei distretti della meccanica $(42,6 \%)$ e quelle localizzate nei distretti del Centro $(45,5 \%)$ e del Nord-Ovest $(45,3 \%)$. Tuttavia, all'aumento della percentuale di imprese distrettuali che ha segnalato un incremento del fatturato nel 2011, corrisponde una crescita altrettanto sensibile della quota di aziende che ha registrato un ridimensionamento del proprio volume d'affari (26\% nel 2011 contro il 19,3\% del 2010).

\subsection{L'export dei distretti 2008-2011}

L'export dei distretti dell'Osservatorio, nel 2009, è stato di 74,6 miliardi di Euro (48,2 miliardi nel settore core business) pari al $25 \%$ della quota export nazionale, con un ridimensionamento rispetto al 2008 (96 miliardi) del 22,3\%.

Dal punto di vista settoriale, i distretti che nel 2009 hanno registrato il calo export più sensibile sono quelli meccanico, arredo-casa e abbigliamento-moda mentre più contenuta è stata la flessione dell'export dei distretti $\mathrm{Hi}$-tech e del comparto degli alimentari-vini.

Sull'andamento export 2009 ha pesato la specializzazione produttiva dei distretti poco presenti nella produzione di beni intermedi (chimica, gomma, plastica, prodotti petroliferi) ed elettronica che, invece, hanno registrato crescita o almeno stabilità nei mercati mondiali. I risultati quindi non riflettono tanto problemi di competitività dei distretti quanto un "effetto domanda mondiale" congiunturalmente meno sensibile ai beni di consumo del sistema moda, mobili ed elettrodomestici (settori di specializzazione dei distretti).

Il 2010, invece, ha rappresentato un anno di forte recupero sul fronte delle esportazioni distrettuali, cresciute dell'11,8\% rispetto ai livelli minimi toccati nel 2009 ed è tornato a crescere anche l'avanzo commerciale dei distretti, che è salito a 42,5 miliardi superando quello dell'intero manifatturiero italiano (cfr. Monitor dei distretti, marzo 2011). L'andamento positivo dell' export è confermato anche per i

competitive sono state quelle che hanno puntato all'innovazione di prodotto e di processo e ad inserirsi in reti commerciali internazionali. 
primi 9 mesi del 2011 quando si è registrato un ulteriore aumento dell'11,3\% rispetto allo stesso periodo del 2010 per un importo di 51,5 miliardi di euro ${ }^{24}$.

I settori dell'automazione-meccanica-gomma e plastica $(+16,4 \%)$, quello dell'abbigliamento-moda $(+12,1)$ quello dell'Hi-tech $(+6,6 \%)$ e quello dell'alimentare-vini $(+5,9 \%)$ continuano a rappresentare il principale traino delle nostre esportazioni mentre a livello geografico sono i 38 distretti del Nord Est a registrare la crescita maggiore $(+12,9 \%)$ seguiti dai 23 distretti del Centro $(+11,4 \%)$ e dai 33 distretti del Nord Ovest $(+10,5 \%)$; in flessione invece l'export dei 7 distretti del Sud $(-3,4 \%)^{25}$. A livello di settore di specializzazione, le criticità del mercato immobiliare continuano a penalizzare il trend dei distretti del sistema-casa, per lo più fermi sui bassi livelli di export del 2010 mentre, a livello geografico, le migliori performance sono registrate dall'Umbria, dal Piemonte e dalla Lombardia, con un aumento tendenziale delle esportazioni nel terzo trimestre 2011 rispettivamente pari al 25,7\%, al 14,1\% e all'11,23\%; più in difficoltà i distretti delle regioni del Sud che, con l'eccezione della Puglia, sono rimaste in territorio negativo, dopo un secondo trimestre 2011 già in calo (cfr. Monitor dei distretti, dicembre 2011).

Per quanto riguarda la destinazione dell'export si rileva una maggiore penetrazione verso i mercati extra UE: per i primi nove mesi del 2011 l'export distrettuale verso i Paesi Ue, pari a 27,7 miliardi di euro, è cresciuto dell' $8,3 \%$ mentre quello verso i Paesi extra Ue, pari a 23,8 miliardi, è aumentato del 15\% (con punte del $21,7 \%$ per i settori automazione-meccanica-gomma-plastica).

\subsection{L'impatto della crisi e la tenuta del modello distrettuale}

La crisi in atto ha fatto emergere segnali di criticità che non solo persistono ma frequentemente si acuiscono determinando un indebolimento dell'organizzazione produttiva di molti distretti:

- l'andamento negativo dell'occupazione. Tra il 2008 e il 2009 nei distretti si sono persi circa 92mila posti di lavoro e la riduzione di addetti ha colpito soprattutto

24 Il dato più confortante è che ben 48 distretti hanno superato i livelli di export registrati nei primi 9 mesi del 2008; tra questi 48 distretti, 11 hanno registrato aumenti dell' export superiori al $20 \%$ con punte del $70 \%$ per le macchine industriali di Treviso, del $60,4 \%$ per il distretto alimentare di Parma e del 31,8\% per la rubinetteria di Omegna.

Nel terzo trimestre del 2011 il livello delle esportazioni distrettuali è inferiore solo del $3,1 \%$ rispetto al corrispondente periodo del 2007 , anno pre-crisi. È interessante rilevare che nei tre trimestri del 2007 i distretti italiani hanno registrato una crescita tendenziale superiore alle esportazioni tedesche manifatturiere $(8,2 \%$ vs $7,5 \%)$. Cfr. Monitor dei Distretti, Intesa Sanpaolo, dicembre 2011.

25 Nei primi 9 mesi del 2011 l'export dei 47 distretti dell'Osservatorio è stato pari a 31,2 miliardi di euro, in crescita del $9,9 \%$ rispetto allo stesso periodo del 2010 . Il dato è sostanzialmente in linea con l'andamento complessivo dei 101 distretti dell'indice Fondazione Edison ma con la differenza che a trainare la crescita export sono stati soprattutto i distretti dell'abbigliamento-moda $(+12,9)$ rispetto a quelli del comparto automazione-meccanica-plastica-gomma $(+11,4 \%)$. 
le piccole imprese ( $\mathrm{da} 10$ a 49 addetti) dove si concentra il $32 \%$ dell'occupazione. Se dall'indagine Unioncamere il quadro del 2011 risulta grave ma con segnali positivi rispetto al 2010 - le aziende che dichiarano di aver ridotto l'organico sono pari al $25,6 \%$ (era il $28 \%$ nel 2010 ) contro il $19 \%$ che rileva un aumento dell'occupazione (12\% nel 2010) - ciò che preoccupa sono i dati 2012: le imprese che hanno previsto un calo dell'occupazione sono pari al $25 \%$ contro soltanto il $6 \%$ che ne prevede un aumento;

- si indebolisce sensibilmente l'interazione tra le imprese, gli enti locali e $i$ soggetti intermedi di rappresentanza. Emergono di frequente segnali di indebolimento del livello di coesione tra i sistemi imprenditoriali e i soggetti intermedi, col rischio di veder affievolirsi la capacità di governance del fenomeno distrettuale rispetto ai cambiamenti dello scenario economico. Gli enti istituzionali vengono giudicati dalle imprese meno dialoganti e lenti nel mettere a punto strumenti che aiutino ad affrontare le difficoltà dell'attuale fase congiunturale. Se i distretti si configurano come luoghi di produzione ma anche come sistemi sociali che necessitano di una manutenzione continua delle competenze e dei valori, agli enti locali gli imprenditori chiedono una reimpostazione della policy che sappia identificare gli interessi del territorio. In concreto, per gli imprenditori risulterebbe strategica una governance unitaria ed efficiente, che garantisca, da un lato, un supporto per la realizzazione di progetti di innovazione e la soluzione di problemi strutturali e, dall'altro lato, favorisca e stimoli azioni virtuose come le aggregazioni tra imprese, l'accesso a nuovi mercati, la progettazione di attività di formazione, l'applicazione di strategie eco-sostenibili;

- le gravi tensioni sui mercati finanziari hanno determinato, per una maggiore prudenza nella valutazione dei rischi, una minore disponibilità delle banche ad erogare finanziamenti e ciò ha prodotto una crisi di liquidità in molte aziende soprattutto quelle di minori dimensioni, che più di altre soffrono l'enorme ritardo nell'incasso dei crediti.

Al sistema bancario s'imputa soprattutto di essere stato eccessivamente prudente pur in un contesto, come quello delle aree distrettuali, in cui si è continuato a produrre, investire e dove si sono registrati i più elevati tassi di esportazione. La crisi di liquidità che ne è scaturita ha in parte minato la capacità propulsiva dei distretti industriali. Se nel 2011 è aumentata la quota di aziende distrettuali che ha dichiarato di aver effettuato nell'anno nuovi investimenti (dal 37,3\% del 2010 al $57,5 \%$ del 2011) e il $33 \%$ prevede di ricorrere al credito per sostenere nuovi investimenti nel 2012, colpisce il fatto che più del $30 \%$ delle aziende di distretto dichiara di aver incontrato difficoltà di accesso al credito negli ultimi sei mesi e che in molti casi tali difficoltà si sostanziano in tassi più onerosi, limitazioni del credito erogato e maggiori garanzie richieste. Il credito bancario risulta essenziale non solo per i nuovi investimenti ma anche per generare flussi di cassa, spesso sotto il livello di sicurezza, anche a causa del sensibile peggioramento negli ultimi due anni dei tempi di pagamento. 
Pur consapevoli degli effetti derivanti dalla grave crisi dei mercati finanziari, si richiede al sistema bancario, da un lato, di rafforzare il rapporto tra territorio e banche locali, che in molti distretti hanno tradizionalmente giocato un ruolo determinante e che negli ultimi tempi a causa di processi di fusioni e incorporazioni hanno ridimensionato la loro originaria vocazione; dall'altro lato di individuare strumenti di valutazione del merito creditizio che riescano a premiare quelle realtà imprenditoriali che vogliono crescere e rafforzarsi, attraverso la realizzazione di progetti di innovazione, l'ingresso in nuovi mercati, l'inserimento in forme reticolari più evolute;

- il passaggio generazionale, l'innalzamento del livello delle competenze all'interno delle aziende, la crescita della cultura d'impresa e i processi di modernizzazione dell'organizzazione aziendale, sono, ancora oggi, variabili critiche che molte imprese distrettuali non riescono a governare con efficacia. In un mercato del lavoro dove le prospettive di nuove assunzioni sono molto limitate persiste, tuttavia, in molte aree produttive la mancanza di personale qualificato e di figure manageriali, che insieme al problema del passaggio generazionale e della limitata cultura aziendale ostacolano lo sviluppo di molte aziende distrettuali. La classe imprenditoriale non è più incarnazione di un animal spirit capace di guidare in modo efficace le principali decisioni ma ha bisogno di crescere culturalmente; la gestione dell'impresa non può basarsi esclusivamente sulla figura dell'imprenditore ma ha bisogno di manager: nel complesso sembra necessario un salto di qualità sebbene un processo di affinamento delle strategie sia stato già messo in atto. In questa prospettiva, le criticità occupazionali dovrebbero rappresentare l'occasione per intensificare, in particolare, il dibattito sugli strumenti che consentano l'adeguamento e il rafforzamento delle competenze professionali, atte a mantenere alta la competitività dei distretti industriali;

- il lavoro sommerso, l'evasione e la concorrenza sleale delle aziende irregolari riducono in maniera distorta la competitività, soprattutto di quelle regolari di minori dimensioni che, anche a causa di questo fenomeno, rischiano l'esclusione dalle filiere produttive. Sullo stesso piano va ricondotto il fenomeno della contraffazione, valutata in una recente ricerca di Confindustria in circa 7 miliardi annui, proveniente per il $70 \%$ dal Far East. Una sua eventuale eliminazione garantirebbe, secondo il Censis, 130mila unità di lavoro aggiuntive nel nostro Paese mentre se si riportasse sul mercato legale il valore della contraffazione stimata si garantirebbe una produzione aggiuntiva per quasi 18 miliardi di euro. Ad essere colpiti sono soprattutto i prodotti Made in Italy e, quindi, soprattutto le produzioni delle aree distrettuali: nel settore dell'occhialeria, ad esempio, la contraffazione tocca punte che comprimono il fatturato del $15 \%$.

Queste criticità, insieme ad altri fenomeni connessi alla globalizzazione che hanno limitato il vantaggio competitivo del territorio, hanno ridimensionato l'effetto distretto per cui la maggiore redditività e produttività generata all'interno delle 
agglomerazioni tende ad affievolirsi se non addirittura ad annullarsi ${ }^{26}$. I vantaggi localizzativi di cui godevano le imprese distrettuali non sono però scomparsi ovunque - vi sono alcuni distretti che registrano ancora oggi performance di gran lunga superiori alla media del settore - e comunque ciò che si rileva positivamente nei numerosi sistemi locali industriali sono la capacità di anticipare con scelte strategiche opportune le tendenze future e le profonde trasformazioni organizzative come risposta alle dinamiche discontinue della congiuntura.

Così come si rileva da un'indagine Unioncamere (Capitolo 2 del Secondo Rapporto), la focalizzazione sulla qualità del prodotto e sulla sua marcata caratterizzazione (design, marchio, posizionamento in specifiche nicchie di mercato) non è più, da tempo, la sola strategia ampiamente diffusa tra le aziende distrettuali, essendo ormai questi asset competitivi acquisiti già prima della fase di crisi della domanda internazionale. Il vero cambiamento degli ultimi anni si sostanzia nell'individuare le strategie più efficaci per vendere i prodotti, soprattutto all'estero.

In una fase di mercato sostanzialmente recessiva ma discontinua, alcuni fenomeni che caratterizzano l'organizzazione produttiva dei distretti appaiono più evidenti di altri:

- si rafforzano le reti di subfornitura che diventano più efficienti e assumono una conformazione a geometria variabile a seconda delle competenze e delle innovazioni delle aziende. Le reti di produzione si stanno allungando andando ben oltre i confini ristretti del territorio distrettuale e la tendenza a creare reti più lunghe è spinta da una duplice esigenza: razionalizzare i costi e acquisire le migliori competenze, ovunque esse si trovino, anche all'estero. Si conferma quindi l'ipotesi che i processi di internazionalizzazione abbiano già trasformato progressivamente i distretti in dis-larghi (Marini, 2012), non annullando il rapporto con il territorio di origine, ma ridefinendolo: il distretto allarga le proprie reti di relazioni, grazie soprattutto alle imprese che hanno internazionalizzato le proprie produzioni, in molti casi trascinando con sé anche i fornitori locali.

La crisi se, da un lato, ha espulso molte imprese marginali, dall'altro lato, ha creato opportunità per quelle imprese che hanno saputo approfittare della possibilità di acquisire asset multifunzionali e di integrare e rafforzare filiere estese internazionalmente. $\mathrm{Al}$ riguardo, è interessante rilevare che nei distretti del tessile-abbigliamento dove si era fatto ricorso intensamente alla delocalizzazione produttiva si registra, soprattutto da parte delle imprese di medie dimensioni, un potenziamento delle filiere localizzate nei territori distrettuali che garantiscono

26 Durante la crisi, la variazione dell' export e del fatturato nei distretti italiani è stata in ogni settore inferiore a quella media italiana. Anche il ritorno alla crescita delle esportazioni che ha caratterizzato il 2010 è stato di minore intensità nei distretti $(12,2 \%)$ rispetto alla media nazionale $(15,0 \%)$. Un'inversione di tendenza si nota invece nei primi tre trimestri del 2011 quando l'export distrettuale è tornato a superare (di circa mezzo punto) la media nazionale, soprattutto per effetto delle buone performance della meccanica e dei sistemi distrettuali nord-occidentali (cfr. Cap. 3 del Terzo Rapporto Osservatorio). 
flessibilità, puntualità delle consegne, co-progettazione e soprattutto riducono al minimo i frequenti errori della produzione delocalizzata: ritardo nelle consegne, ordini inevasi, necessità di riparazioni.

I criteri di selezione dei partner sembrano mutare: oltre all'affidabilità del fornitore e alla rapidità di consegna diventa sempre più importante la capacità del partner di progettare insieme al committente. Inoltre, tendono a svilupparsi, grazie alle opportunità offerte dalla nuova disciplina, le reti formali e le più frequenti sono quelle realizzate con università e centri di ricerca. Il distretto, inoltre, si sta dimostrando uno degli ambienti più congeniali per la formalizzazione di cooperazioni, fino ad oggi spontanee, mediante il contratto di rete previsto dalla legge n. 33/2009;

- si moltiplicano le forme di innovazioni non più esclusivamente di prodotto e di processo ma sempre più frequentemente legate ai servizi offerti ai clienti finali. In particolare, i fattori critici di successo sembrano risiedere nella cura per il dettaglio, negli elevati standard qualitativi, nella capacità di progettazione e nel miglioramento delle strategie commerciali e distributive. È evidente che questa carica innovativa è presente soprattutto nelle aziende di maggiori dimensioni e in posizione di leadership all'interno del distretto mentre appare più sfumata nelle aziende minori che presidiano solo poche fasi lungo la filiera. Oggi, se si considera il tema dell'innovazione, all'interno dei distretti è possibile riconoscere diverse tipologie di imprese: quelle leader che si caratterizzano per la capacità di proiettare le proprie relazioni oltre i confini del distretto (e quindi di accedere a fonti esterne di conoscenza); quelle co-operative sub-contractors che non competono nei mercati esterni, ma collaborano con quelle leader nella produzione e nell'innovazione; le follower, che sfruttano le opportunità locali senza contribuire ai processi di generazione dell'innovazione e le unlinked, marginali e che occupano segmenti a minor remuneratività e con minori barriere all'entrata, esposte quindi alla concorrenza di altri sub-fornitori, anche esteri, con costi del lavoro più bassi (Marini e Toschi, 2011).

Nonostante questa eterogeneità, il carattere distintivo dei distretti continua a risiedere nella capacità di amalgamare ruoli differenti e generare nel contempo processi produttivi e organizzativi caratterizzati da un elevato grado di innovazione. La focalizzazione sul prodotto e la rispondenza alle aspettative del mercato finale resta il paradigma interpretativo dominante delle strategie competitive dei distretti. L'innovazione si sostanzia anche in nuove forme organizzative dei processi produttivi, caratterizzate da continue modifiche strategiche con mix variabili di processi di esternalizzazione e internalizzazione della produzione. Da questo punto di vista, molte imprese di distretto mostrano una capacità di generare e adattare alle proprie esigenze reti lunghe e corte, creando sistemi di circolazione della conoscenza e di collaborazione ormai flessibili e destrutturati ${ }^{27}$;

27 In questa prospettiva, è emblematico il caso del distretto della maglieria e dell'abbigliamento di Carpi (Intesa Sanpaolo, Monitor dei distretti, Marzo 2010), che 
- si diffonde la cultura della responsabilità sociale e si investe in processi produttivi eco-sostenibili. L'omogeneità delle produzioni, del livello tecnologico e delle scelte gestionali rendono il distretto un ambito favorevole per perseguire strategie e politiche ambientali in chiave competitiva. I distretti dell'Osservatorio hanno una conoscenza robusta dei temi legati alla responsabilità sociale, con una maggiore consapevolezza da parte di quelli dell'abbigliamento moda e dell'automazione meccanica. Per il mondo distrettuale il punto di forza della responsabilità sociale è la tutela ambientale e in subordine la governance e le politiche verso i dipendenti; le motivazioni che spingono le aziende ad adottare pratiche di responsabilità sociale sono il miglioramento delle relazioni con gli stakeholder interni (dipendenti e management), l'aumento dell'efficienza e il rafforzamento della reputazione aziendale mentre risultano meno importanti il vantaggio commerciale e l'incentivo fiscale/pubblico.

A conferma di questa tendenza vi sono i dati dell'Indagine Unioncamere dove si rileva che più di un terzo delle aziende distrettuali ha realizzato o realizzerà a breve investimenti in tecnologie verdi (era il $24 \%$ nel 2010): per il 53,8\% i nuovi investimenti verdi riguardano impianti e tecnologie per la riduzione dei consumi energetici, per il 30,5\% modifiche sugli impianti al fine di ridurre l'impatto ambientale e per il $15,7 \%$ realizzazione di prodotti ecocompatibili;

- cresce la propensione all'esportazione e si intensifica l'accesso e il presidio dei mercati extra Ue. Nel 2011 e in particolare nel secondo trimestre è ulteriormente cresciuta la propensione a esportare nei "nuovi" mercati ad alto potenziale, dove i distretti hanno registrato un aumento dell'export intorno al 13,6\% tendenziale. Molto positiva ancora la performance conseguita in Cina (606 milioni di euro esportati vs 483 milioni nello stesso periodo del 2010). È confermata anche la ripartenza dell'altro "motore" emergente, la Russia $(+20,6 \%)$ e ha iniziato ad acquisire un ruolo maggiore anche un altro BRIC, il Brasile, dove le esportazioni, guidate dai distretti della meccanica, hanno raggiunto la quota di 173 milioni di euro.

dopo una fase di crisi negli anni Novanta, ha registrato negli anni Duemila una sensibile crescita del fatturato. La ripresa del distretto è stata il frutto di un intenso processo di riposizionamento strategico: orientamento verso segmenti medio-alti del mercato (meno esposti alla concorrenza di prezzo), investimenti nella ricerca stilistica del prodotto, ampliamento della gamma fino ad offrire un total look, affermazione di marchi propri, controllo diretto dei canali distributivi. Effetti di questo riposizionamento strategico sono stati la concentrazione del fatturato in poche aziende, la chiusura di molte imprese marginali, un valore della produzione del comparto confezione superiore a quello della maglieria (specializzazione tradizionale del distretto). Sull'evoluzione del distretto ha pesato il ruolo delle imprese leader, che hanno avuto il merito di creare reti produttive in grado di effettuare l'intero processo (dalla creazione del modello alla consegna nel canale di vendita) con la partnership di sub-fornitori selezionati capaci di co-progettare e disponibili a partecipare al rischio degli investimenti. 


\section{II fenomeno reti nei distretti}

La consapevolezza di non essere autosufficienti e la necessità di superare i limiti posti dai vincoli di proprietà hanno indotto molte aziende di dimensioni ridotte ad affiancare alle forme classiche di gestione meccanismi di regolazione, come le relazioni di cooperazione, sotto forma di reti, che in questi ultimi anni hanno di fatto rappresentato il vantaggio competitivo di alcune imprese. Queste forme di collaborazione strategica tendono sempre più a diffondersi e, in alcuni casi, arrivano addirittura alla condivisione di investimenti e dei relativi rischi mediante la specializzazione dei processi produttivi: un unico processo viene ripartito in più fasi ed ogni operatore in base alle proprie competenze distintive si occupa di una specifica fase, generando in tal modo economie di scala e di apprendimento.

La rete può rappresentare una delle migliori soluzioni al problema dimensionale del nostro sistema economico in quanto si è osservato che quando l'impresa leader acquisisce la consapevolezza di non poter fare a meno dei partner, inizialmente formalizza i rapporti mediante contratti e successivamente acquisisce il controllo delle imprese nodali trasformando la rete in un gruppo di imprese.

Generalmente in un distretto operano imprese indipendenti, integrate in una rete di relazioni di cooperazione informale e di lungo periodo. Da questo punto di vista, il distretto rappresenta indubbiamente un terreno fertile per il sorgere di un sistema reticolare, tenuto conto che si innesta in un tessuto di relazioni preesistenti in aree geograficamente ben delimitate. Quello che non sempre accade è che i rapporti di comunanza geografica si evolvano in rapporti di comunanza gestionale. Pertanto, "il distretto e le aree-sistema non costituiscono condizione né necessaria né sufficiente per l'applicabilità dei reticoli evoluti di cooperazione" (Faedo e Farinet, 1999, p. 49). Tuttavia, grazie alla maggiore interazione sociale, alle comuni radici culturali, alla circolazione agevolata delle competenze e delle conoscenze, i distretti possono costituire degli acceleratori del processo di sviluppo di reti evolute.

La rete, dentro e fuori il distretto, rappresenta ancora un valore, capace di contribuire positivamente al miglioramento della competitività delle singole imprese partner. Molto saldi appaiono i legami funzionali tra imprese all'interno dei distretti, specie nel caso delle reti di produzione, che progressivamente sono divenute più complesse. Se è vero, così come si rileva dall'indagine Unioncamere (cfr. Capitolo 2 del Secondo Rapporto), che per una parte molto consistente delle imprese (37\%) i principali fornitori sono ancora «di prossimità», quote altrettanto consistenti (31\%) indicano la presenza di fornitori nella regione di localizzazione dell'impresa e fuori dalla regione $(26,7 \%)$, così come per più del $5 \%$ i fornitori sono collocati all'estero. $\mathrm{Si}$ configurano in tal modo reti lunghe e corte allo stesso tempo, un sistema policentrico, con molti nodi, espressione della complessità delle nuove e diversificate esigenze dei distretti industriali italiani. Ci sono reti molto accentrate su un soggetto leader, generalmente una media impresa di successo del Made in Italy e reti orizzontali che condividono un progetto di prodotto o commerciale ma anche finalizzate a condividere conoscenze e aggiornamento continuo. 
Il sistema delle reti distrettuali si è notevolmente evoluto nel corso dei decenni, di pari passo con l'innalzamento progressivo e costante della qualità dei prodotti realizzati. In particolare, diventa sempre più sofisticato il processo di selezione dei fornitori e sempre più frequenti sono $\mathrm{i}$ casi di co-progettazione tra committente $\mathrm{e}$ fornitore. Al riguardo, l'indagine Unioncamere rileva che solo il $30 \%$ degli intervistati valuta un fornitore in primo luogo in base al prezzo mentre per il resto interviene un mix di altri criteri come, ad esempio, la qualità delle forniture, la rapidità di consegna della merce, i rapporti consolidati, la capacità del fornitore di progettare o di apportare miglioramenti ai prodotti. Altre forme di collaborazione si profilano all'interno dei distretti: dai legami con i centri universitari di ricerca (prevalentemente localizzati nella provincia del distretto) ai consorzi per l'export e per l'internazionalizzazione a quelli per l'acquisto in comune di materie prime. Si tratta di schemi di rete assai diffusi, il più delle volte provvisori, quindi con un tasso di turnover elevato. Sotto questo profilo, tendono a diffondersi anche relazioni tra imprese localizzate in distretti diversi e tra imprese di settori di specializzazione differenti.

La crisi ha rimesso in gioco gran parte della forza di coesione intra-distrettuale, ma per il momento l'architettura complessiva, quel legame forte tra territorio e impresa, sembra mostrare ancora molti punti di forza. Occorre tuttavia chiedersi quanto le reti distrettuali siano attuali. Se da un lato esse garantiscono il perpetuarsi di un modello che resiste alle difficoltà più evidenti, dall'altro è sempre più evidente che tali reti debbano aprirsi all'esterno. L'allargamento costante delle reti collaborative dovrebbe essere una priorità essenziale per i distretti: debordare dai confini del localismo ed allargare la visuale potrebbe, dunque, essere un nuovo importante obiettivo di crescita. Il distretto, in sostanza, può essere la base attraverso cui far passare reti sempre più ampie e nuove, forme di collaborazione diverse: dai consorzi acquisti a quelli per l'export, da quelli per la sperimentazione di innovazioni fino alle piattaforme logistiche comuni.

Nel 2009, in Italia è stata approvata la legge che disciplina le reti di imprese (L. 33/2009 poi modificata con L. 122/2010). Per le imprese aderenti ad una rete viene prevista la possibilità di sottoscrivere un "contratto di rete" teso a formalizzare la posizione preesistente. Con tale contratto due o più imprese si obbligano ad esercitare in comune una o più attività economiche rientranti nei rispettivi oggetti sociali allo scopo di accrescere la reciproca capacità innovativa e la competitività sul mercato $^{28}$.

28 Finora, in Italia sono stati stipulati 523 contratti di rete che coinvolgono 2.807 imprese, di cui 646 situate in Lombardia, 443 in Toscana, 312 in Emilia Romagna e 238 in Veneto. La maggior parte dei contratti (369, pari al 70\% del totale contratti) vede coinvolte imprese operanti nella stessa regione, mentre sono 105 i contratti che insistono su 2 regioni e 49 quelli che coinvolgono imprese con sede in 3 o più regioni. Con riferimento al macrosettore di attività economica delle imprese aderenti, circa il $41 \%$ opera nell'industria in senso stretto, il $34 \%$ nel settore dei servizi, il $10 \%$ nell'edilizia/costruzioni. 
Nessuna contrapposizione o esclusione, quindi, tra reti e distretti, bensì uno strumento in linea con l'esigenza di maggiore flessibilità. Le recenti esperienze testimoniano che la necessità di porre in attuazione progetti fortemente innovativi determina una spinta alla condivisione dei progetti tra aziende della stessa filiera, a volte collocate al di fuori del proprio territorio distrettuale, che resta comunque la culla di esperienze industriali, di conoscenze tecniche, di specifiche conoscenze manifatturiere. Il territorio, inoltre, può e deve mettere a disposizione strumenti e soggetti di supporto allo sviluppo: non è la territorialità il tema da superare, bensì la funzione del territorio che va riposizionata. In questa prospettiva, un ruolo fondamentale di stimolo allo sviluppo di queste forme organizzative lo rivestono le associazioni di categoria e soprattutto le Camere di commercio.

Queste istituzioni, nell'attuale fase delicata del ciclo di vita dei distretti, dovrebbero svolgere alcune funzioni di importanza prioritaria ${ }^{29}$ : riuscire a conservare e consolidare sul territorio il patrimonio di know-how produttivo oggi a rischio, sia per l'uscita dal mercato degli addetti che detengono queste professionalità sia per lo scarso interesse delle giovani generazioni ad acquisire queste competenze ${ }^{30}$; implementare nuovi circuiti di conoscenze che permettano un efficace e rapido trasferimento tra chi le crea e chi le utilizza; favorire una partnership con le banche anche al fine di migliorare i criteri di valutazione del rischio che tengano conto delle peculiarità delle imprese distrettuali.

Infine, sarebbe auspicabile la costituzione di reti tra distretti. L'evoluzione del modello distrettuale, infatti, è rappresentata dallo sviluppo di partnership tra distretti diversi ma caratterizzati da una stretta interconnessione delle vocazioni produttive. Le aziende che operano nello stesso settore o in settori affini hanno necessità comuni e insieme possono realizzare progetti mirati ad aumentarne la competitività sui mercati internazionali.

In questa prospettiva si colloca il Progetto di collaborazione Verona-Puglia, avviato da circa due anni, che punta a realizzare forme di collaborazione stabile e duratura nel tempo tra aziende delle aree veronese e pugliese per supplire alla carenza di lavorazioni specifiche, anche in seguito alla chiusura di molti subfornitori locali. Si tratta, di fatto, di una delocalizzazione della produzione in house, invece che nell'Europa dell'Est, che prende le mosse da un «atto di legalità», nell'ultima parte del 2008, quando il prefetto di Verona face chiudere per irregolarità i laboratori cinesi che rifornivano i semilavorati alle imprese del Consorzio Verona

29 Un sistema territoriale caratterizzato da istituzioni e centri di servizio che costituiscono un vero punto di riferimento e sostegno per gli imprenditori si ritrova nel sistema delle macchine agricole di Modena e Reggio Emilia, con una quota export di circa il $60 \%$ del fatturato. Le iniziative promosse dalle istituzioni locali, in termini di servizi e assistenza, hanno contribuito, secondo il giudizio degli imprenditori, all'internazionalizzazione delle imprese e alla loro capacità d'innovazione.

30 Nel distretto tessile di Carpi la collaborazione tra istituzioni e imprese ha dato vita alla costituzione del Campus della Moda, dove vengono insegnate competenze stilistiche e manageriali con l'obiettivo, riuscito, di mantenere nel territorio il patrimonio di professionalità e competenze produttive. 
Moda. Si cercarono quindi nuovi fornitori in Serbia, Slovacchia, Romania ma senza successo. In particolare, i subfornitori di quelle aree territoriali non garantivano la qualità e soprattutto i tempi di consegna. A questo punto, anche grazie all'intervento della Federazione dei Distretti Italiani, che ha tra gli obiettivi la creazione di progettualità sinergiche tra i distretti, venne creato un contatto tra il Distretto Veneto dell'abbigliamento VeronaModa e il Distretto Filiera ModaPuglia. Il progetto aveva partner autorevoli come Confindustria Bari e Confartigianato Puglia nonché il Gruppo Bancario Monte dei Paschi. Ad oggi, si è raggiunto il risultato di avere prodotti di più elevata qualità a costi lievemente superiori senza i disagi connessi all'internazionalizzazione della strategia. In definitiva, il Progetto Verona-Puglia rappresenta un esempio di "rete nazionale tra distretti", un'esperienza che, se replicata per altre aree sistema e per settori di specializzazione, potrebbe garantire un notevole incremento del valore aggiunto prodotto nel nostro Paese. La collaborazione, infatti, prevede un ulteriore modello di cooperazione per competere su scala globale, finalizzato in particolare alla integrazione delle imprese lungo la filiera della moda, allo sviluppo di prodotti di nicchia e di campionari di total look per le varie fasce di mercato. Sono anche allo studio metodi per sviluppare modelli di comarketing per la formazione congiunta di marchi, per realizzare campagne pubblicitarie e per organizzare la distribuzione dei prodotti su scala globale, creando così un'integrazione completa di servizi.

Nell'ambito della promozione di reti tra distretti si colloca anche il Progetto Elica, che ha l'obiettivo di incentivare la collaborazione tra aziende distrettuali innovative, italiane e olandesi, operanti nei settori dell'high tech, del design e dell'agroalimentare. La denominazione "Elica" enfatizza l'accelerazione dell'innovazione e delle opportunità di business di cui beneficiano i soggetti coinvolti nei progetto, accelerazione resa possibile dalla stretta cooperazione tra aziende, governi e istituzioni universitarie e di ricerca: le tre pale dell'elica. Per i promotori di questo progetto, Elica è orientata a interconnettere attivamente cluster $\mathrm{e}$ aziende innovative, competitive e aperte al cambiamento nella convinzione che le aziende operanti nei cluster olandesi e nei distretti italiani nei settori dell'high tech, del design e dell'agroalimentare possano beneficiare fortemente di tali interscambi e, operando congiuntamente, espandere i relativi mercati.

\section{Conclusioni}

Dai tre Rapporti dell'Osservatorio Nazionale Distretti Italiani emerge un quadro in movimento, fatto di territori in cui la produzione organizzata in filiere lunghe o corte e il sistema delle reti collaborative assumono ancora un valore profondo, ma nel quale si inseriscono con crescente evidenza dei fenomeni di frattura rispetto al passato: alcuni positivi che favoriscono il riposizionamento del sistema distrettuale nel contesto produttivo nazionale, altri negativi che rischiano di compromettere equilibri consolidati e di determinare una crisi irreversibile del "territorio". 
Le criticità esaminate, insieme ad altri fenomeni connessi alla globalizzazione che hanno limitato il vantaggio competitivo del territorio, hanno ridimensionato l'effetto distretto per cui la maggiore redditività e produttività generata all'interno delle agglomerazioni tende ad affievolirsi se non addirittura ad annullarsi. I vantaggi localizzativi di cui godevano le imprese distrettuali non sono però scomparsi ovunque - vi sono alcuni distretti che registrano ancora oggi performance di gran lunga superiori alla media del settore - e comunque ciò che si rileva positivamente nei numerosi sistemi locali industriali sono la capacità di anticipare con scelte strategiche opportune le tendenze future e le profonde trasformazioni organizzative come risposta alle dinamiche discontinue della congiuntura.

Tuttavia, ciò che si rileva dall'analisi dei dati e dei fenomeni è ancora una volta la capacità dei distretti non solo di resistere ad una fase recessiva permanente ma anche di anticipare le tendenze e di rappresentare un modello di riferimento per le modalità di interrelazione e collaborazione tra imprese, per la propensione a investire, per l'accesso a nuovi mercati e per la vocazione alla sostenibilità.

Forte è la sensazione che la crescita non risieda solo in nuovi investimenti in innovazione ma in un cambiamento culturale che parta, in primo luogo, dalla classe imprenditoriale e che sia debitamente accompagnato e alimentato dai principali soggetti intermedi operanti nella dimensione locale.

C'è da chiedersi dunque in che misura e come i distretti produttivi vadano ripensati.

La questione è urgente e dalle scelte che ne conseguono dipendono in parte gli esiti del nostro sistema industriale, caratterizzato più di altri dalle agglomerazioni industriali: alla metà degli anni 2000 circa il $60 \%$ dei 27 milioni di occupati manifatturieri dell'UE a 15 era concentrato in Germania (28\%), Italia (13\%), Francia (12\%) ma oltre la metà degli addetti delle agglomerazioni industriali dei tre Paesi era concentrato in Italia con un'incidenza delle agglomerazioni dell'industria italiana (in termini di addetti) tre volte superiore alla Germania e dieci volte superiore alla Francia (Alampi et al., 2012, p. 38).

In uno scenario di mercato globalizzato, il ruolo del territorio paradossalmente diventa ancora più importante per sostenere la stessa sopravivenza dei distretti. C'è bisogno, tuttavia, di un territorio «attrezzato», nel quale le relazioni fra le imprese e fra queste ed i centri di servizio si caratterizzino per capacità progettuali. Il territorio diventa vantaggio competitivo nella misura in cui mette a disposizione delle imprese conoscenze, technicalities avanzate, servizi finanziari innovativi. La circolazione di conoscenze deve svilupparsi non solo tra imprese ma anche intessendo rapporti con Università, centri di ricerca, poli tecnologici per sostenere l'ulteriore terziarizzazione dei distretti produttivi. La storia degli ultimi anni mostra come in alcuni casi la rete tra imprese e tecnostrutture ha funzionato mentre in altri casi si è rilevata meno efficace. Occorre, pertanto, riflettere su come migliorare l'operatività dei legami tra imprese e strutture di servizio.

Il futuro di molti distretti dipenderà dalla loro capacità di conservare il ricco patrimonio di conoscenze e saperi produttivi. Oggi, anche per via della bassa propensione delle nuove generazioni ad occuparsi di attività produttive/artigianali 
considerate poco remunerative e prestigiose, è sempre meno possibile basare la formazione del capitale umano su processi spontanei (Intesa Sanpaolo, 2009). Per evitare il rischio concreto che si disperdano competenze professionali/artigianali "secolari" è necessario potenziare e promuovere strategicamente le scuole tecniche locali nonché stimolare l'interesse delle nuove generazioni verso quelle specifiche attività produttive ${ }^{31}$.

In questa prospettiva, risulta prioritario ripensare alla governance dei distretti che attualmente rappresenta il loro principale punto debole: una governance che sappia interpretare ma soprattutto anticipare le esigenze delle imprese e che offra soluzioni concrete per migliorarne la competitività.

A tal fine, occorre agire su alcune leve strategiche: governance, cooperazione, innovazione, finanza. In questa fase congiunturale delicatissima, i distretti non possono essere lasciati soli: ogni Paese ha il dovere di salvaguardare le proprie eccellenze e i distretti, oggi, rappresentano una delle eccellenze produttive dell'Italia.

Pertanto, come afferma Becattini, "in un Paese come il nostro la politica economica dovrebbe preoccuparsi anzitutto di traghettare oltre la crisi $i$ nostri distretti industriali, che, come dimostra chiaramente la nostra bilancia dei pagamenti, da un lato costituiscono il motore principale dell'economia italiana, dall'altro danno un grande contributo alla coesione sociale del Paese" (Primo Rapporto Osservatorio, p. 15).

\section{Bibliografia}

ALAMPI D., CONTI L., IUZZOLINO G., MELE D. (2012), "Le agglomerazioni industriali italiane: peculiarità strutturali nel confronto internazionale", in Atti del Convegno Banca d'Italia Le trasformazioni dei sistemi produttivi locali, Università di Bologna, 31 gennaio e $1^{\circ}$ febbraio 2012.

ALTER C., HAGE J. (1993), Organizations working together, Sage Publications, London.

BANCA D'ITALIA (2009), Bollettino statistico, IV trimestre.

BECATTINI G. (1979), "Dal settore industriale al distretto industriale. Alcune considerazioni sull'unità di indagine nell'economia aziendale", Economia e Politica Industriale, n. 1, pp. 7-21 (pubblicato anche in Becattini G., Industrial districts. A new approach to industrial change, Edward Elgar, 2004).

BECATTINI G. (1989), Modelli locali di sviluppo, Il Mulino, Bologna.

31 Al riguardo, è interessante l'esperienza del distretto meccanico di Lecco dove si rileva una forte sensibilità alla formazione e al consolidamento delle competenze dello specifico settore. In particolare, è stato avviato il Progetto Labomet che prevede formazione specifica per i processi produttivi della meccanica mediante l'erogazione di corsi di aggiornamento post diploma, corsi di specializzazione e riqualificazione professionale, corsi di aggiornamento e formazione per i docenti. Il progetto, promosso dal Comitato di Distretto e supportato dai Gruppi Metalmeccanici e Scuola delle Associazioni di Categoria, nasce dall'esigenza diffusa di coprire un differenziale formativo che non risulta adeguato ai bisogni espressi dalle imprese del comparto metalmeccanico. 
BECATTINI G. (1991), "Il distretto industriale marshalliano come concetto socioeconomico", in Pyke F., Becattini G., Sengenberger W., (a cura di), Distretti industriali e cooperazione tra imprese in Italia, Quaderno di "Studi e Informazioni" della Banca Toscana, n. 34, pp. 51-65.

BECATTINI G. (1999), "La fioritura delle piccole imprese e il ritorno dei distretti industriali", Economia e Politica Industriale, n. 103, pp. 5-16 (Ristampato in Becattini G., Il distretto industriale, Rosenberg \& Sellier, Torino, 2000, pp. 25-37).

BECATTINI G. (2007), Il Calabrone Italia, Il Mulino, Bologna.

BECATTINI G. (2009), "Traghettiamo i nostri distretti industriali oltre la crisi", Il Ponte, giugno n. 6, pp. 94-96.

BECATTINI G., BELLANDI M., DE PROPRIS L. (2009) (a cura di), A Handbook of Industrial Districts, Edward Elgar, Cheltenham UK.

BECATTINI G., RULLANI E. (1996), "Local systems and global connections: the role of knowledge", in Cossentino F., Pyke F., Sengenberger W., Local and Regional Response to Global Pressure. The Case of Italy and its Industrial Districts, International Institute for Labour Studies, Geneva.

BELLANDI M., RUSSO M. (1994), Distretti industriali e cambiamento economico locale, Rosenberg \& Sellier, Torino.

BELUSSI F., SAMMARRA A. (2009) (a cura di), Business Networks in Clusters and Industrial Districts. The governance of the global Value chain, Routledge, London.

BENTIVOGLI C., GALLO M. (2011), "Nord Est: metamorfosi di un modello", in L'economia del Nord Est, Banca d'Italia, Seminari e convegni - Workshops and Conferences, n. 8, Ottobre.

BRENNER T. (2004), Local Industrial Clusters: Existence, Emergence \& Evolution, Routledge, London.

BRESCHI S., MALERBA F. (2005), Clusters, Networks, and Innovation, Oxford University Press, Oxford.

CONTRACTOR F.J., LORANGE P. (2002), Cooperative strategies and alliances, Business \& Economics, Oxford.

DEI OTTATI G. (2009), "An industrial district facing the challenges of globalisation: Prato today", European Planning Studies, vol. 17, n. 12, pp. 1817-1835.

FAEDO P., FARINET A. (1999), "Modelli reticolari evolutivi e strategie di cooperazione tra piccole e medie imprese: alcune ipotesi interpretative", Economia e Politica Industriale, n. 104, pp. 35-56.

FAZIO V., RICCIARDI A. (2008), Il distretto della Pesca di Mazara del Vallo. Una buona pratica di cooperazione tra aziende internazionali, Franco Angeli, Milano.

FEDERAZIONE DEI DISTRETTI ITALIANI (a cura di), Rapporto Osservatorio Nazionale dei Distretti Italiani, anni vari, disponibile su www.osservatoriodistretti.org.

FORESTI G., GUELPA F., PALUMBO A. (2011), "Analisi economico-finanziaria dei distretti industriali italiani”, III Rapporto Osservatorio Nazionale dei Distretti Italiani.

FORTIS M., QUADRIO CURZIO A. (2003), "Distretti: sì ai marchi d'origine”, Il Sole 24 Ore, 23 aprile 2003.

GALLO M. (2011), "Alla ricerca della competitività: il cambiamento strategico nelle imprese industriali del Nord Est", in L'economia del Nord Est, Banca d'Italia, Seminari e convegni - Workshops and Conferences n. 8, Ottobre 2011.

INTESA SANPAOLO (2009), "Economia e Finanza dei Distretti industriali", Rapporto n. 2, pp. 1-165, dicembre.

INTESA SANPAOLO (2010), Monitor dei Distretti, marzo.

INTESA SANPAOLO (2011), Monitor dei Distretti, marzo. 
INTESA SANPAOLO (2011), Monitor dei Distretti, dicembre.

ISTAT (2005), I Distretti Industriali. $8^{\circ}$ Censimento generale dell'industria e dei servizi 2001, 16 dicembre.

IZZO F., RICCIARDI A. (2006), Relazioni di cooperazione e reti di imprese. Il caso della Campania, Franco Angeli, Milano.

IUZZOLINO G. (2011), "Le recenti trasformazioni nei distretti industriali italiani", in Federazione dei Distretti Italiani (a cura di), III Rapporto Osservatorio Nazionale dei Distretti Italiani, pp. 83-109, disponibile sul sito http://www.osservatoriodistretti.org

MARINI, D. (2012), Innovatori di confine. I percorsi del nuovo Nord Est, Marsilio, Venezia.

MARINI D., TOSCHI G. (2011), "Imprese distrettuali e processi di innovazione", Federazione dei Distretti Italiani (a cura di), III Rapporto Osservatorio Nazionale dei Distretti Italiani, pp. 249-261, disponibile sul sito http://www.osservatoriodistretti.org

ONIDA F., VIESTI G., FALZONI A.M. (1992) (a cura di), I distretti industriali: crisi o evoluzione?, Egea, Milano.

PASTORE P. (2008), "La governance nei distretti produttivi italiani”, Amministrazione \& Finanza, vol. 11, pp. 29-39.

PASTORE P. (2009), "Modelli e strutture di governance nei distretti industriali e nelle reti di imprese", in Tommaso S., Distretti e reti di imprese. Evoluzione organizzativa, finanza innovativa, valutazione mediante rating, Franco Angeli, Milano.

PASTORE P. (2010), "Modelli di governance nelle reti di imprese", Amministrazione \& Finanza, vol. 7, pp. 32-40.

PASTORE P., TOMMASO S. (2012), "Relazioni tra governance e performance economicofinanziarie nei distretti industriali", in Mosconi F., (a cura di), Metamorfosi del Modello Emiliano: c'è ancora un futuro per i distretti industriali?, Il Mulino, Bologna.

PIORE M.J. (2009), "Conceptualizing the dynamics of industrial districts", in Becattini G., Bellandi M., De Propis L., (a cura di), A Handbook of Industrial Districts, Edward Elgar, Cheltenham UK.

PIORE M.J., SABEL C. (1984), The second industrial divide. Possibilities for prosperity, Basic Books, New York.

RICCIARDI A. (2003), Le reti di imprese. Vantaggi competitivi e pianificazione strategica, F. Angeli, Milano.

RICCIARDI A. (2006), "Distretti industriali: criticità della gestione finanziaria e strumenti innovativi", in Antoldi F., (a cura di), Piccole imprese e distretti industriali, Il Mulino, Bologna.

RICCIARDI A. (2008-a), "I distretti industriali: motore dello sviluppo economico del nostro Paese", Amministrazione \& Finanza, Inserto, n.18, pp. I-XV

RICCIARDI A. (2008-b), "Polo aeronautico campano", in AIP - Associazione Italiana Politiche Industriali (a cura di), Reti di impresa oltre $i$ distretti. Nuove forme di organizzazione produttiva, di coordinamento e di assetto giuridico, Il Sole 24 Ore Libri, Milano.

RICCIARDI A. (2009), "Consorzio della Moda della provincia di Verona", in AIP Associazione Italiana Politiche Industriali (a cura di), Fare reti d'impresa. Dai nodi distrettuali alle maglie lunghe: una nuova dimensione per competere, Il Sole 24 Ore Libri, Milano.

RICCIARDI A., PASTORE P. (2010), Outsourcing strategico, Franco Angeli, Milano.

RICCIARDI A. (2010-a), "Le Pmi localizzate nei distretti industriali: vantaggi competitivi, evoluzione organizzativa, prospettive future", Quaderni di ricerca sull'artigianato, maggio, n. 54, pp. 129-177. 
RICCIARDI A. (2010-b), “L'impatto della crisi sui distretti industriali: tendenze evolutive e scenari futuri", Il Ponte, n. 4, pp. 51-62.

RICCIARDI A. (2010-c), "Strategie di cooperazione tra aziende e mitigazione del rischio operativo: i vantaggi competitivi delle reti di imprese", in Airoldi G., Brunetti G., Corbetta G., Invernizzi G., (a cura di), Economia Aziendale \& Management: scritti in onore di Vittorio Coda, Università Bocconi Editore, Milano.

RICCIARDI A. (2011), "Le aziende dei distretti della meccanica: evidenze emerse dalle analisi di bilancio", Perfomance \& Management, n. 2, pp. 15-23.

RICCIARDI A. (2012), "I distretti tra crisi e ripresa: i risultati del III Rapporto (2011) dell'Osservatorio Nazionale Distretti Italiani”, in Mosconi F., (a cura di), Metamorfosi del Modello Emiliano: c'è ancora un futuro per i distretti industriali?, Il Mulino, Bologna.

RULLANI E. (2002), "Il distretto industriale come sistema adattivo complesso", in Quadrio Curzio A., Fortis M., (a cura di), Complessità e distretti industriali. Dinamiche, modelli, casi reali, Il Mulino, Bologna.

RUSSO A. (2012), Politiche pubbliche tra globalizzazione e spazio locale, Carocci editore, Roma.

SANCETTA G. (1997), I gruppi di imprese minori, Cedam, Padova.

STABER U. (2001), "The structure of Networks in Industrial Districts", International Journal of Urban and Regional Research, vol. 25, n. 3, pp. 537-552.

TOMMASO S. (2009-a), Distretti e Reti di imprese. Evoluzione organizzativa, finanza innovativa, valutazione mediante rating, Franco Angeli, Milano.

TOMMASO S. (2009-b), "I distretti produttivi: analisi dei recenti provvedimenti normativi", Amministrazione \& Finanza, n. 8, pp. 26-32.

UNICREDIT CORPORATE BANKING, FEDERAZIONE DEI DISTRETTI ITALIANI (a cura di) (2009), I Distretti della Meccanica-10 realtà a confronto per valorizzare le eccellenze ed agire sui territori.

UNIONCAMERE-TAGLIACARNE (2006), I tassi di interesse a livello provinciale, 15 dicembre.

UNIONCAMERE, FONDAZIONE SYMBOLA (2011), GreenItaly. L'economia verde sfida la crisi, Rapporto 2011, disponibile sul sito http://www.unioncamere.gov.it

VIESTI G. (2000), Come nascono i distretti industriali, Laterza, Roma-Bari. 